\title{
Exact Hausdorff and packing measures for random self-similar code-trees with necks
}

\author{
Sascha Troscheit \\ Department of Pure Mathematics, University of Waterloo, Waterloo, Ont., N2L 3G1, Canada \\ stroscheit@uwaterloo.ca
}

April 1, 2019

\begin{abstract}
Random code-trees with necks were introduced recently to generalise the notion of $V$-variable and random homogeneous sets. While it is known that the Hausdorff and packing dimensions coincide irrespective of overlaps, their exact Hausdorff and packing measure has so far been largely ignored. In this article we consider the general question of an appropriate gauge function for positive and finite Hausdorff and packing measure. We first survey the current state of knowledge and establish some bounds on these gauge functions. We then show that self-similar code-trees do not admit a gauge functions that simultaneously give positive and finite Hausdorff measure almost surely. This surprising result is in stark contrast to the random recursive model and sheds some light on the question of whether $V$-variable sets interpolate between random homogeneous and random recursive sets. We conclude by discussing implications of our results.
\end{abstract}

\section{Contents}

1 Introduction and Random Models $\quad 2$

1.1 Random code-trees and their attractors . . . . . . . . . . . . . . . 2

2 Hausdorff and packing measure of random attractors $\quad 6$

2.1 Exact Hausdorff and packing measure for random recursive constructions . . 7

3 Bounds on the gauge function for random homogeneous constructions 9

3.1 Equicontractive homogeneous random attractors . . . . . . . . . . . 9

4 The non-existence of gauge functions for self-similar code-trees with necks 14

4.1 Equicontractive homogeneous random attractors . . . . . . . . . . . . . . . . 14

4.2 Gauge functions for random code-trees with necks . . . . . . . . . . . 18

5 Exact packing measure $\quad 22$

5.1 Bounds for equicontractive RIFS . . . . . . . . . . . . . . . . . 23

5.2 Existence of a gauge function . . . . . . . . . . . . . . . . . . . 24

6 Implications for a random implicit theorem 24

6.1 The implicit theorems . . . . . . . . . . . . . . . . . . . 25

$6.2 V$-variable interpolation . . . . . . . . . . . . . . . 26 
The Hausdorff dimension and measure of random constructions such as branching processes, Brownian motion and stochastically self-similar sets has been studied since the 1980s and much progress has been made on conditions for positive and finite Hausdorff and packing measure. We refer the reader to the seminal work of Athreya [AN72] on Branching processes and Watanabe [Wat07], Liu [Liu00] and [Liu96] for recent progress on the Hausdorff and packing measure of Galton-Watson processes. The related stochastically self-similar sets were analysed by Graf, Williams, and Mauldin [GMW88], [MGW87]; Berlinkov and Mauldin [BM02]; and Berlinkov [Ber03]; and we will come back to those in Section 2. Apart from these processes, the question of exact Hausdorff and packing measures was answered for some random re-orderings by $\mathrm{Hu}[\mathrm{Hu} 95]$ and [Hu96], and for self-avoiding walks on the Sierpiński Gasket by Hattori [Hat00]. For deterministic sets, Olsen [Ols03] considered the exact Hausdorff measure on some Cantor sets.

Despite this great canon of work, random homogeneous attractors have largely been ignored, even though they represent a very natural model for random sets. In this article we remedy this gap by giving bounds on the exact gauge functions required and showing that there is no gauge function which simultaneously gives positive and finite Hausdorff measure; a stark contrast to all other examples just mentioned. We start by defining random codetrees in a similar spirit to the seminal papers by Järvenpää et al. [JJK ${ }^{+} 14$, JJLS16, JJWW17] in Section 1, and provide historical context to our results in Section 2.

Since the model of random code trees is fairly abstract we continue in Section 3 by reducing the model to random homogeneous attractors with equal contraction ratios. These equicontractive homogeneous attractors are simpler to study and we first state gauge function that give a finer quantification of the Hausdorff dimension and then prove that there cannot be a gauge function that gives positive and finite Hausdorff measure almost surely. This is followed by a statement and proof of the general theorem for self-similar random code-trees with necks in Section 4.2.

We end this article by proving some analogous results for the packing measure in Section 5 and discuss wider implications of our result in Section 6.

\section{Introduction and Random Models}

Let $k \in \mathbb{N}$, and let $\Lambda \subset \mathbb{R}^{k}$ be a non-empty compact set. We will use $\Lambda$ to index our random choice of iterated function systems and associate with it a Borel probability measure $\mu$ compactly supported on $\Lambda$. For $\lambda \in \Lambda$ let $\mathbb{I}_{\lambda}=\left\{f_{\lambda}^{1}, f_{\lambda}^{2}, \ldots, f_{\lambda}^{\mathcal{N}_{\lambda}}\right\}$ be a collection of $\mathcal{N}_{\lambda} \in \mathbb{N}$ contracting similarities on $\mathbb{R}^{d}$, i.e. maps that satisfy $\left|f_{\lambda}^{i}(x)-f_{\lambda}^{i}(y)\right|=c_{\lambda}^{i}|x-y|$ for some $c_{\lambda}^{i} \in(0,1)$. Finally, let $\mathbb{L}=\left\{\mathbb{I}_{i}\right\}_{i \in \Lambda}$ be a (not necessarily finite) collection of iterated function systems with at most $\mathcal{N}$ similarities. We will refer to the pair $(\mathbb{L}, \mu)$ as a random iterated function system (RIFS). Unless otherwise noted we assume

$$
2 \leq \mathcal{N}:=\sup _{\lambda \in \Lambda} \mathcal{N}_{\lambda}<\infty \text { and } 0<c_{\min }:=\inf _{\lambda \in \Lambda} \min _{1 \leq i \leq \mathcal{N}_{\lambda}} c_{\lambda}^{i} \leq \sup _{\lambda \in \Lambda} \max _{1 \leq i \leq \mathcal{N}_{\lambda}} c_{\lambda}^{i}=: c_{\max }<1 .
$$

\subsection{Random code-trees and their attractors}

\subsubsection{The general model}

Consider the rooted $\mathcal{N}$-ary tree. The general idea of random code-trees is achieved by 'randomly' picking a labelling function $\tau$ that labels each node with a single $\lambda \in \Lambda$, chosen

Mathematical Subject Classification 2010: 28A78; 28A80, 37C45, 60J80.

Keywords: random code-trees, exact Hausdorff measure, packing measure, gauge functions, selfsimilarity, dimension theory. 
according to some probability measure $\mathbb{P}$. We first describe the general set-up, before talking about specific methods of picking the function $\tau$.

We denote the space of all possible functions (and hence labellings) of the full tree by $\mathcal{T}$ and refer to individual realisations by $\tau \in \mathcal{T}$. In this full tree we address vertices by which branch was taken; if $v$ is a node at level $k$ we write $v=\left(v_{1}, v_{2}, \ldots, v_{k}\right)$, with $v_{i} \in\{1, \ldots, \mathcal{N}\}$ and root node $v=($.$) . We write \Sigma_{k}$ for the nodes at level $k$ and $\Sigma^{*}=\bigcup_{k \in \mathbb{N}_{0}} \Sigma_{k}$ for the set of all nodes, where $\mathbb{N}_{0}=\mathbb{N} \cup\{0\}$ and $\Sigma_{0}=\{()$.$\} . Thus,$

$$
\Sigma^{*}=\{\{(.)\},\{(1),(2), \ldots,(\mathcal{N})\},\{(1,1),(1,2), \ldots,(1, \mathcal{N}),(2,1), \ldots,(\mathcal{N}, \mathcal{N})\}, \ldots\} .
$$

We slightly abuse notation and consider $\tau$ both as a function $\tau: \Sigma^{*} \rightarrow \Lambda$, where $\tau(v) \in \Lambda$ and as a labelled full tree. Given a node $v \in \Sigma^{*}$ we define the shift $\sigma^{v} \tau$ to be the full subtree starting at vertex $v$, with $\sigma^{(\cdot)} \tau=\tau$. At this point we note that since $\Lambda$ was a compact topological space, the set of all realisations $\mathcal{T}$ is also a compact topological space with respect to the obvious product topology by Tychonoff's theorem.

We write $e_{\lambda}^{j}$ for the letter representing the map $f_{\lambda}^{j} \in \mathbb{I}_{\lambda}$. For each labelled full tree $\tau$, we construct another rooted labelled $\mathcal{N}$-ary tree $\mathbf{T}_{\tau}$, where each node is labelled by a 'coding' describing a composition of maps. Given two codings $e_{1}$ and $e_{2}$, we write $e_{1} e_{2}=e_{1} \odot e_{2}$ for concatenation. We let $\varepsilon_{0}$ be the empty word and use the symbol $\emptyset$ as a multiplicative zero, i.e. $\emptyset \odot e=e \odot \emptyset=\emptyset$, to represent the empty function. Similarly, if $\left\{e_{1}, \ldots, e_{n}\right\}$ is a collection of codings, then $\left\{e_{1}, \ldots, e_{n}\right\} \cup \emptyset=\left\{e_{1}, \ldots, e_{n}\right\}$. This letter $\emptyset$ is used to 'delete' a subbranch if the number of maps in an IFS is less than $\mathcal{N}$.

Definition 1.1. Let $\mathbf{T}_{\tau}$ be a labelled tree, we write $\mathbf{T}_{\tau}(v)$ for the label of node $v$ of the tree $\mathbf{T}_{\tau}$. The code-tree $\mathbf{T}_{\tau}$ is then defined inductively:

$$
\mathbf{T}_{\tau}((.))=\varepsilon_{0} \text { and } \mathbf{T}_{\tau}(v)=\mathbf{T}_{\tau}\left(\left(v_{1}, \ldots, v_{k}\right)\right)=\mathbf{T}_{\tau}\left(\left(v_{1}, \ldots, v_{k-1}\right)\right) \odot e_{\tau\left(v_{k-1}\right)}^{v_{k}}
$$

for $1 \leq v_{k} \leq \mathcal{N}_{\tau\left(v_{k-1}\right)}$ and $e_{\tau\left(v_{k-1}\right)}^{v_{k}}=\emptyset$ otherwise. We refer to the the set of all codings at the $k$-th level by

$$
\mathbf{T}_{\tau}^{k}=\bigcup_{v \in \Sigma_{k}} \mathbf{T}_{\tau}(v)
$$

We can now define the attractor of the code-tree.

Definition 1.2. Let $\mathbb{L}$ be a collection of IFS and let $\tau \in \mathcal{T}$. The attractor of a code-tree $F_{\tau}$ is the compact set satisfying

$$
F_{\tau}=\bigcap_{k=1}^{\infty} \bigcup_{e \in \mathbf{T}_{\tau}^{k}} f_{e_{1}} \circ f_{e_{2}} \circ \cdots \circ f_{e_{k}}(\Delta),
$$

where $\Delta$ is a sufficiently large compact set, satisfying $f_{\lambda}^{i}(\Delta) \subseteq \Delta$ for all $\lambda \in \Lambda$ and $1 \leq i \leq$ $\mathcal{N}_{\lambda}$.

This general - and somewhat abstract - way of describing geometric objects is very flexible. For example, let $\Lambda=\{0,1,2,3\}, f_{l}(x)=x / 2, f_{r}(x)=x / 2+1 / 2$ and set $\mathbb{I}_{0}=\{\}$, $\mathbb{I}_{1}=\left\{f_{l}\right\}, \mathbb{I}_{2}=\left\{f_{r}\right\}$, and $\mathbb{I}_{3}=\left\{f_{l}, f_{r}\right\}$. Then, $\mathbf{T}_{\tau}^{k}$ represents subsets of all dyadic intervals of length $2^{-k}$ and $F_{\tau}$ is the limsup set of a sequence of decreasing dyadic intervals. Therefore, constructing $\tau$ in the appropriate way, we can recover every compact subset of the unit interval with a code-tree.

Instead of constructing code-trees with a certain set in mind, we could also choose $\tau$ at random. In the above example, choosing each $\mathbb{I}_{i}$ with probability $1 / 4$ at every step in the construction gives rise to Mandelbrot percolation of the unit line, an example of stochastically self-similarity. We now describe the main ways of choosing $\tau$. 


\subsubsection{The random recursive measure}

Random recursive attractors are random sets that exhibit a stochastic self-similarity. They were first investigated in the 1980s by Falconer [Fal86] and Graf [Gra87] and we will summarise their and later results in Section 2. These random fractals satisfy the following equality (in distribution), where $\lambda$ is chosen according to some compactly supported Borel probability $\mu$ on $\Lambda$.

$$
F_{\tau}={ }_{d} \bigcup_{i=1}^{\mathcal{N}_{\lambda}} f_{\lambda}^{i}\left(F_{\tau}\right)
$$

There exists a natural measure $\mathbb{P}_{T}$ on the collection of code-trees, induced by $\mu$ which describes the same model. We avoid giving a description here, and briefly comment that $\mathbb{P}_{T}$ can be obtained by choosing $\tau$ such that for every open set $\mathcal{O} \subseteq \Lambda$, the probability that $\tau(v) \in \mathcal{O}$ is $\mu(\mathcal{O})$ for every $v \in \Sigma^{*}$. Further, given distinct $v, w \in \Sigma^{*}$ and (not necessarily distinct) open sets $\mathcal{O}_{v}, \mathcal{O}_{w} \subseteq \Lambda$, the probability that $\tau(v) \in \mathcal{O}_{v}$ and $\tau(w) \in \mathcal{O}_{w}$ are independent.

\subsubsection{The homogeneous measure}

Another natural measure, $\mathbb{P}_{H}$, is obtained by choosing an iterated function system of $\mathbb{L}$ according to $\mu$ at every level $k$ of the construction and applying the same random IFS to all nodes at level $k$. While the IFS is still chosen i.i.d. with respect to the tree levels, all nodes at the same level share the same label. This is why it is called the homogeneous measure.

For this model one does not need the full abstract model of random code-trees and we will use the following, somewhat simpler, notation. Consider each IFS $\mathbb{I}_{\lambda}$ as a self-map on compact subsets of $\mathbb{R}^{d}$. That is $\mathbb{I}_{\lambda}: \mathcal{K}\left(\mathbb{R}^{d}\right) \rightarrow \mathcal{K}\left(\mathbb{R}^{d}\right)$, given by $\mathbb{I}_{\lambda}(K)=\bigcup_{f \in \mathbb{I}_{\lambda}} f(K)$. We will index random realisations by an infinite sequence with entries in $\Lambda$. The set of realisations, denoted by $\Omega$, is given by $\Omega=\Lambda^{\mathbb{N}}$ and realisations $\omega \in \Omega$ are chosen according to the product (probability) measure $\mathbb{P}_{H}=\mu^{\mathbb{N}}$.

Definition 1.3. The $k$-level coding with respect to realisation $\omega=\omega_{1} \omega_{2} \cdots \in \Omega=\Lambda^{\mathbb{N}}$ is

$$
\mathbf{C}_{\omega}^{k}=\bigcup_{1 \leq j_{i} \leq \mathcal{N}_{\omega_{i}}} e_{\omega_{1}}^{j_{1}} e_{\omega_{2}}^{j_{2}} \ldots e_{\omega_{k}}^{j_{k}}(k \in \mathbb{N}) \quad \text { and } \quad \mathbf{C}_{\omega}^{0}=\varepsilon_{0}
$$

The set of all finite codings $\mathbf{C}_{\omega}^{*}$ is defined by

$$
\mathbf{C}_{\omega}^{*}=\bigcup_{i=0}^{\infty} \mathbf{C}_{\omega}^{i}
$$

Definition 1.4. The $k$-level prefractal $F_{\omega}^{k}$ and the random homogeneous random attractor $F_{\omega}$ are

$$
F_{\omega}^{k}=\mathbb{I}_{\omega_{1}} \circ \mathbb{I}_{\omega_{2}} \circ \cdots \circ \mathbb{I}_{\omega_{k}}(\Delta)=\bigcup_{e \in \mathbf{C}_{\omega}^{k}} f_{e_{1}} \circ f_{e_{2}} \circ \cdots \circ f_{e_{k}}(\Delta)
$$

and

$$
F_{\omega}=\bigcap_{k=1}^{\infty} F_{\omega}^{k}=\bigcap_{k=1}^{\infty} \bigcup_{e \in \mathbf{C}_{\omega}^{k}} f_{e_{1}} \circ f_{e_{2}} \circ \cdots \circ f_{e_{k}}(\Delta),
$$

where $\Delta \in \mathcal{K}\left(\mathbb{R}^{d}\right)$ is such that $f_{\lambda}^{i}(\Delta) \subseteq \Delta$ for all $\lambda \in \Lambda$ and $1 \leq i \leq \mathcal{N}_{\lambda}$. 


\subsection{4 $V$-variable sets and random code-trees with necks}

$V$-variable sets were first introduced by Barnsley et al. [BHS05, BHS08, BHS12] and are characterised by allowing up to $V \in \mathbb{N}$ different structures at every level of the construction, see also Freiberg [Fre10] for a recent survey. A more general model was developed by Järvenpää et al. [JJK ${ }^{+} 14$, JJLS16, JJWW17] in the context of self-affine maps with random translates and is the model that we will adopt in this manuscript. We note that setting this model up in the right way allows us to recover both $V$-variable and random homogeneous attractors.

The central property that was crucial for the proofs in both the $V$-variable and the codetree setting was the almost sure existence of necks. Informally, these necks are levels in the construction at which point all subtrees are identical. Thus, these models still possess some homogeneity which is exploited in proofs.

Definition 1.5. Let $\mathcal{T}$ be the space of all mappings $\tau: \Sigma^{*} \rightarrow \Lambda$. Let $\mathbf{N}=\left(N_{1}, N_{2}, \ldots\right) \in \mathbb{N}^{\mathbb{N}}$ be a strictly increasing sequence of integers such that

$$
\sigma^{v} \tau=\sigma^{w} \tau \quad \text { for all } v, w \in \Sigma_{N_{k}} \text { that satisfy } \mathbf{T}(v), \mathbf{T}(w) \neq \emptyset .
$$

We say that $N_{k}$ is a neck level and that $\mathbf{N}$ is a neck list.

All that is left to describe is a measure of how individual relations are to be picked. Here we consider a very general approach and all that we require are some properties of the measure with respect to a dynamical system on $\left(\mathcal{T}, \mathbb{N}^{\mathbb{N}}\right)$ we call the neck shift.

Definition 1.6. Let $(\tau, \mathbf{N}) \in\left(\mathcal{T} \times \mathbb{N}^{\mathbb{N}}\right)$, where $\mathbf{N}$ is a strictly increasing sequence of natural numbers. We define the neck shift $\Pi:\left(\mathcal{T} \times \mathbb{N}^{\mathbb{N}}\right) \rightarrow\left(\mathcal{T} \times \mathbb{N}^{\mathbb{N}}\right)$ by

$$
\Pi(\tau, \mathbf{N})=\left(\sigma^{1_{N_{1}}(\tau)} \tau,\left(N_{2}(\tau)-N_{1}(\tau), N_{3}(\tau)-N_{1}(\tau), \ldots\right)\right),
$$

where $1_{N_{1}}$ is the node at level $N_{1}$ consisting solely of $1 \mathrm{~s}$.

Whereas $\mathbb{P}$ was the product measure for random homogeneous systems above, we now only require $\mathbb{P}$ to be an ergodic $\Pi$-invariant Borel probability measure such that the first neck has finite expectation and necks are independent.

Definition 1.7. Let $(\tau, \mathbf{N}) \in\left(\mathcal{T} \times \mathbb{N}^{\mathbb{N}}\right)$, where $\mathbf{N}$ is a strictly increasing sequence of natural numbers, and let $\Pi$ be the neck shift. A code-tree measure is any $\Pi$-invariant Borel measure on $\left(\mathcal{T} \times \mathbb{N}^{\mathbb{N}}\right)$ such that

$$
\mathbb{E}\left(N_{1}\right)=\int_{\left(\mathcal{T} \times \mathbb{N}^{\mathbb{N}}\right)} N_{1}(\tau) d \mathbb{P}(\tau)<\infty
$$

and for all open ${ }^{1}$ subsets $A \subseteq\left(\mathcal{T} \times \mathbb{N}^{\mathbb{N}}\right)$,

$$
\begin{aligned}
\mathbb{P}\{(\tau, \mathbf{N}) \in A\}=\mathbb{P}\{\Pi(A)\} \cdot \mathbb{P}\left\{(\tau, \mathbf{N}) \in\left(\mathcal{T} \times \mathbb{N}^{\mathbb{N}}\right) \mid \exists\left(\tau^{\prime}, \mathbf{N}\right) \in\right. \text { A such that } \\
\left.\qquad \tau(v)=\tau^{\prime}(v) \text { for all } v \in \bigcup_{j=1}^{N_{1}(\tau)} \Sigma_{j}\right\},
\end{aligned}
$$

\footnotetext{
${ }^{1}$ The topology here is the product topology of the previously stated topology of $\mathcal{T}$ with the discrete topology on $\mathbb{N}^{\mathbb{N}}$.
} 
We note that condition (1.1) guarantees independence between neck levels, which further implies strong mixing and ergodicity of the neck shift. Without loss of generality we assume that all $(\tau, \mathbf{N}) \in\left(\mathcal{T}, \mathbb{N}^{\mathbb{N}}\right)$ have infinitely many necks, since this set has full measure with respect to the neck measure $\mathbb{P}$.

Clearly, the shift map $\sigma$ on $\Omega$ for random homogeneous attractors satisfies the conditions of a neck measure and so does the natural measure for $V$-variable sets. In Section 4.2 we will prove that this model does not admit any gauge function but we will first consider simple reductions of this model.

We end by referring the reader to [RU11] and [Tro17b] for other approaches using random graphs which overlap with this model to some extend.

\section{Hausdorff and packing measure of random attractors}

We often have to assume some conditions on the overlaps of images in the iterated function systems to state meaningful dimension theoretic results. In this article we will make use of the uniform open set condition, but remark that some of the quoted results require slightly different overlap conditions.

Definition 2.1 (uniform open set condition (UOSC)). Let $\mathbb{L}=\left\{\mathbb{I}_{\lambda}\right\}_{\lambda \in \Lambda}$ be a collection of IFSs. We say that $\mathbb{L}$ satisfies the uniform open set condition (UOSC) if there exists an open set $\mathcal{O}$ such that

$$
f_{\lambda}^{i}(\mathcal{O}) \subseteq \mathcal{O} \text { and } f_{\lambda}^{i}(\mathcal{O}) \cap f_{\lambda}^{j}(\mathcal{O})=\varnothing \text { for all } \lambda \in \Lambda \text { and } 1 \leq i, j \leq \mathcal{N}_{i} \text { where } i \neq j .
$$

One can easily determine the almost sure Hausdorff dimension of these random attractors if one assumes the uniform open set condition and similarity maps. Recall that $c_{\lambda}^{i}$ is the contraction rate of $f_{\lambda}^{i} \in \mathbb{I}_{\lambda}$. The Hausdorff (and packing) dimension of random homogeneous attractors is given, almost surely, by the unique $s$ satisfying

$$
\exp \mathbb{E}\left(\log \sum_{j=1}^{\mathcal{N}_{\omega_{1}}}\left(c_{\omega_{1}}^{j}\right)^{s}\right)=1,
$$

see e.g. [Ham92, RU11, Tro17b]. For random recursive sets the almost sure Hausdorff dimension is the unique $s$ satisfying

$$
\mathbb{E}\left(\sum_{j=1}^{\mathcal{N}_{\omega_{1}}}\left(c_{\omega_{1}}^{j}\right)^{s}\right)=1,
$$

see e.g. [Fal86, Gra87]. To ease notation we write $\mathfrak{S}_{\lambda}^{s}=\sum_{j=1}^{\mathcal{N}_{\lambda}}\left(c_{\lambda}^{j}\right)^{s}$ for $\lambda \in \Lambda$ and note that we assume

Condition 2.2. Let $(\mathbb{L}, \mu)$ be a random iterated function system. We assume that there exists $\mathcal{N}$ such that $\mathcal{N}_{\lambda} \leq \mathcal{N}$ for all $\lambda \in \Lambda$ and there exist $0<c_{\min } \leq c_{\max }<1$ such that $c_{\min } \leq c_{i}^{j} \leq c_{\max }$ for all $i \in \Lambda$ and $j \in\left\{1, \ldots, \mathbb{I}_{i}\right\}$. For the random recursive model we further assume $\mathbb{E}\left(\mathfrak{S}_{\lambda}^{0}\right)>1$ and for the random homogeneous model we assume $\mathbb{E}\left(\log \mathfrak{S}_{\lambda}^{0}\right)>0$.

We note that Condition 2.2 implies that $c_{\min }^{s} \leq \mathfrak{S}_{\lambda}^{s}<\mathcal{N}$ and $s \log c_{\min } \leq \log \mathfrak{S}_{\lambda}^{s}<$ $\log \mathcal{N}$ for all $s \geq 0$. We immediately obtain that $\mathbb{E}\left(\mathfrak{S}_{\tau_{1}}^{s}\right)<\infty$ (random recursive) and $\mathbb{E}\left(\log \mathfrak{S}_{\omega_{1}}^{s}\right)<\infty$ (random homogeneous) for all $s \geq 0$. Note that under these conditions we also have $\operatorname{Var}\left(\log \mathfrak{S}_{\omega_{1}}^{s}\right)<\infty$ for all $s \geq 0$ for the random homogeneous model. 
Definition 2.3. A random iterated function system $(\mathbb{L}, \mu)$ is called almost deterministic if there exists $s$ such that $\mathfrak{S}_{\lambda}^{s}=1$ for $\mu$-almost every $\lambda \in \Lambda$.

If such $s$ exists it must necessarily be the almost sure Hausdorff dimension, i.e.

$$
s=\operatorname{ess}_{\tau \in \mathcal{T}} \operatorname{dim}_{H}\left(F_{\tau}\right) .
$$

Proposition 2.4 (Graf [Gra87]). Let $(\mathbb{L}, \mu)$ be a random iterated function system satisfying the UOSC and Condition 2.2 with associated random recursive set $F_{\tau}$ and write $s_{0}=\operatorname{ess}_{\operatorname{dim}_{H}} F_{\tau}$. If $(\mathbb{L}, \mu)$ is almost deterministic then

$$
0<\mathscr{H}^{s_{0}}\left(F_{\tau}\right)<\infty \quad \text { (a.s.) }
$$

and $\mathscr{H}^{s_{0}}\left(F_{\tau}\right)=0$ (a.s.) otherwise.

For random homogeneous attractors an analogous result holds. This is a special case of the one considered in [RU11].

Proposition 2.5 (Roy and Urbanski [RU11]). Let $(\mathbb{L}, \mu)$ be a random iterated function system satisfying the UOSC and Condition 2.2 with associated random homogeneous set $F_{\omega}$ with almost sure Hausdorff dimension $s_{0}=\operatorname{ess}_{\operatorname{dim}_{H}} F_{\omega}$. If $(\mathbb{L}, \mu)$ is almost deterministic then

$$
0<\mathscr{H}^{s_{0}}\left(F_{\omega}\right)<\infty \quad \text { (a.s.) }
$$

and $\mathscr{H}^{s_{0}}\left(F_{\omega}\right)=0$ (a.s.) otherwise.

In fact, more is known. If an attractor is not almost deterministic the packing measure of the random homogeneous attractor $F_{\omega}$ and the random recursive attractor $F_{\tau}$ is infinite almost surely, see Roy and Urbanski [RU11] and Berlinkov and Urbanski [BM02], respectively.

\subsection{Exact Hausdorff and packing measure for random recursive constructions}

Recall that a gauge function $h: \mathbb{R}_{0}^{+} \rightarrow \mathbb{R}_{0}^{+}$is a left-continuous, non-decreasing function such that $h(r) \rightarrow 0$ as $r \rightarrow 0$. If there exists a constant $\lambda>1$ such that for all $x>0$ we have $h(2 x) \leq \lambda h(x)$ we say that $h$ is doubling. Recall the definition of the $h$-Hausdorff measure.

Definition 2.6. Let $F \subseteq \mathbb{R}^{d}$ and let $h$ be a gauge functions. The $h$-Hausdorff $\delta$-premeasure of $F$ is

$$
\mathscr{H}_{\delta}^{h}(F)=\inf \left\{\sum_{k=1}^{\infty} h\left(\left|U_{k}\right|\right) \mid\left\{U_{i}\right\} \text { is a countable } \delta \text {-cover of } F\right\},
$$

where the infimum is taken over all countable $\delta$-covers. The $h$-Hausdorff measure of $F$ is then

$$
\mathscr{H}^{h}(F)=\lim _{\delta \rightarrow 0} \mathscr{H}_{\delta}^{h}(F) .
$$

Note that the gauge function need only be defined and non-decreasing on $\left[0, r_{0}\right]$ for some $r_{0}>0$ since we are only concerned in its limit as $r \rightarrow 0$. Without loss of generality we shall assume that $h(t) \leq \bar{h}:=\min \{1, \sup h(s)\}$ where the supremum is taken over the largest interval where $h$ is defined and non-decreasing. Further we set $h(t)=\bar{h}$ for all $t>r_{0}$. For example, by writing $h(t)=t \log \log (1 / t)$ we mean

$$
h(t)=\left\{\begin{array}{ll}
\log t \log \log (1 / t) & \text { for } t \leq r_{0} \\
r_{0} \log \log \left(1 / r_{0}\right) & \text { for } t>r_{0}
\end{array},\right.
$$


where $r_{0}$ is the unique solution to $\log \log \left(1 / r_{0}\right)=\left(\log \left(1 / r_{0}\right)\right)^{-1}$, its unique stationary point.

For the random recursive case, Graf, Mauldin, and Williams determined the natural gauge that gives positive and finite Hausdorff measure.

Theorem 2.7 (Graf, Mauldin, and Williams [GMW88, MGW87]). Let (L, $\mu$ ) be a random iterated function system that is not almost deterministic. Let $F_{\tau}$ be the associated random recursive attractor. Assume that

$$
\mathbb{E}\left(\sum_{j}\left(c_{\omega_{1}}^{j}\right)^{0}\right)>1
$$

Let

$$
h_{\beta}^{s}(t)=t^{s}(\log \log (1 / t))^{1 / \beta} \text { and } \beta_{0}=\sup \left\{\beta \mid \sum_{j}\left(c_{\omega_{1}}^{j}\right)^{s /(1-1 / \beta)} \leq 1(\text { a.s. })\right\} .
$$

Then, $\mathscr{H}^{h_{\beta}^{s_{0}}}\left(F_{\tau}\right)<\infty$ for all $\beta>\beta_{0}$, where $s_{0}=\operatorname{ess}_{\operatorname{dim}_{H}} F_{\tau}$.

The authors then proceed to give technical conditions under which $\beta_{0}=1-s / d$, where $d$ is the dimension of the ambient space. Under these conditions the $h_{\beta_{0}}^{s}$-Hausdorff measure of $F_{\tau}$ is positive and finite almost surely. Checking the conditions one obtains that Mandelbrot percolation of $[0,1]^{d}$ has positive and finite measure at this critical value $\beta_{0}$.

Liu [Liu00] investigated the Gromov boundary of Galton-Watson trees with i.i.d. randomised descendants. Let $m=\mathbb{E}(N)$, where $N$ is the number of descendants, $\alpha=\log m$, and assume that $\mathbb{E}(N \log N)<\infty$. If $\bar{m}=\operatorname{ess} \sup N<\infty$, then the appropriate gauge function for which one obtains positive and finite measure of the boundary (with respect to a natural metric) is

$$
h(t)=t^{\alpha}(\log \log (1 / t))^{\beta}, \text { where } \beta=1-\frac{\log m}{\log \bar{m}} .
$$

For the packing measure to be positive and finite the appropriate gauge function is

$$
h^{*}(t)=t^{\alpha}(\log \log (1 / t))^{\beta^{*}}, \text { where } \beta^{*}=1-\frac{\log m}{\log \underline{m}},
$$

with $\underline{m}=\operatorname{ess} \inf N>1$

Berlinkov and Mauldin [BM02] provide the following, more general result for the packing measure of random recursive sets. Under the same almost deterministic condition they show that the $s$-dimensional packing measure is positive and finite almost surely. When this fails, the packing measure is $\infty$ almost surely, assuming the UOSC in both cases. Let $s$ denote the almost sure packing dimension. The authors prove that for the gauge function

$$
h_{\beta}^{s}(t)=t^{s}(\log \log (1 / t))^{\beta}, \text { where } \beta \text { satisfies } 0<\liminf _{a \rightarrow 0}-a^{-1 / \beta} \log \mathbb{P}_{T}\left(\mathfrak{S}_{\lambda}^{s}<a\right)<\infty,
$$

the packing measure is almost surely finite. We remark that the constant $\beta$ may not exist and only coincides with the $\beta_{0}$ in the Hausdorff measure statement in trivial cases.

Additionally, Berlinkov and Mauldin give an integral test [BM02, Theorem 6] to determine whether the packing measure is 0 almost surely. They further conjecture a lower bound that Berlinkov proved in [Ber03]: If the random variable $\mathfrak{S}_{\lambda}^{s}$ is of exponential type, i.e. if

$$
C^{-1} a^{1 / \beta} \leq-\log \mathbb{P}_{T}\left(0<\mathfrak{S}_{\lambda}^{s} \leq a\right) \leq C a^{1 / \beta}
$$

for some $C, \beta>0$ and all $a \in(0,1)$, then the packing measure is positive and finite almost surely with gauge function $h_{\beta}^{s}(t)$. 


\section{Bounds on the gauge function for random homoge- neous constructions}

It is of course of interest to determine the gauge functions for which one obtains positive and finite measure for random homogeneous systems. In particular, self-similar and selfconformal sets that satisfy the open set condition have positive and finite Hausdorff measure. One might expect that random homogeneous are of a similar nature and that a gauge function of the form $t^{s}(\log \log (1 / t))^{\beta}$ for some exponent $\beta$ should work for all natural random code-tree constructions.

However, we will show that this turns out not to be the case. Indeed, for $s=F_{\omega}$, we first prove better bounds on the fine dimension, i.e. bounds on $h$ that give positive or finite measure. In the next Section we show that there is no gauge function that gives positive and finite measure, but the bounds established here are still of separate interest. We will argue that

$$
h_{1}(t)=t^{s} \exp (\sqrt{(\log (1 / t))(\log \log \log (1 / t))}),
$$

gives good bounds on the positivity and finiteness of random homogeneous constructions. Let $\beta, \gamma \in \mathbb{R}$, we similarly define

$$
h_{1}(t, \beta, \gamma)=t^{s} \exp \left(\sqrt{2 \beta(\log (1 / t))\left(\log \log \log \left(1 / t^{\beta}\right)\right)}\right)^{1-\gamma}
$$

\subsection{Equicontractive homogeneous random attractors}

The first thing to note is that $h_{1}(t, \beta, \gamma)$ is doubling in $t$.

Lemma 3.1. Fix $\beta, \gamma>0$. There exists $t_{0}, \rho>0$ such that

$$
h_{1}(t, \beta, \gamma) \leq \rho h_{1}(2 t, \beta, \gamma) \leq \rho^{2} h_{1}(t, \beta, \gamma)
$$

for all $0<t<t_{0}$.

Proof. Let $\kappa \in \mathbb{R}$ and

$$
h_{*}(x+\kappa)=\sqrt{\beta(x+\kappa) \log \log (\beta(x+\kappa))} .
$$

This is well defined for $\log \log \beta(x+\kappa)>1 \Longrightarrow x>e^{e} / \beta-\kappa$. It can easily be seen that this function is strictly increasing in $x$, and differentiating we obtain,

$$
h_{*}^{\prime}(x+\kappa)=\sqrt{\beta} \cdot \frac{1 /(\log (\beta(x+\kappa))+\log \log (\beta(x+\kappa))}{2 \sqrt{(x+\kappa) \log \log (\beta(x+\kappa))}} .
$$

Then, for $\kappa>0$,

$$
\begin{aligned}
\frac{h_{*}^{\prime}(x)}{\sqrt{\beta}} & =\frac{1 /(\log (\beta x)+\log \log (\beta x)}{2 \sqrt{x \log \log (\beta x)}} \\
& >\frac{1 /(\log (\beta(x+\kappa))+\log \log (\beta(x+\kappa))}{2 \sqrt{x \log \log (\beta(x+\kappa))}}=\frac{h_{*}^{\prime}(x+\kappa)}{\sqrt{\beta}}
\end{aligned}
$$

and so $h_{*}^{\prime}(x+\kappa)-h_{*}^{\prime}(x)<0$ and $h_{*}(x+\kappa)-h_{*}(x)$ is decreasing, i.e. there exists some $\rho_{0}$ such that

$$
0 \leq h_{*}(x+\kappa)-h_{*}(x) \leq \rho_{0}
$$


Now substituting $\kappa=-\log 2$ and $x=-\log t$, i.e. $x+\kappa=\log (1 / 2 t)$, we obtain, for $0<t<t_{0}$ and $t_{0}>0$ small enough,

$$
0 \leq \sqrt{\beta \log (1 /(2 t) \log \log (\beta \log 1 /(2 t))}-\sqrt{\beta \log (1 / t) \log \log (\beta \log 1 / t)} \leq \rho_{0},
$$

and

$$
\begin{aligned}
2^{s} & \leq \frac{(2 t)^{s}}{t^{s}} \cdot e^{(1-\gamma) \sqrt{2} \cdot(\sqrt{\beta \log (1 /(2 t) \log \log (\beta \log 1 /(2 t))}-\sqrt{\beta \log (1 / t) \log \log (\beta \log 1 / t)})} \\
& \leq 2^{s} e^{(1-\gamma) \sqrt{2} \rho_{0}} .
\end{aligned}
$$

But then

$$
2^{s} \leq \frac{h_{1}(2 t, \beta, \gamma)}{h_{1}(t, \beta, \gamma)} \leq 2^{s} e^{(1-\gamma) \sqrt{2} \rho_{0}}
$$

as required.

We require some further results on homogeneous systems satisfying the UOSC. Let $\varepsilon>0$ and $\mathcal{O}$ be the open set guaranteed by the UOSC. We define $\Xi_{\varepsilon}(\omega)$ be the words in $\mathbf{C}_{\omega}^{*}$ such that $\left|f_{e}(\mathcal{O})\right| \leq \varepsilon$, but $\left|f_{e^{-}}(\mathcal{O})\right|>\varepsilon$ for all $e=e_{1} e_{2} \ldots e_{k} \in \Xi_{\varepsilon}(\omega)$, where $e^{-}=e_{1} e_{2} \ldots e_{k-1}$.

Lemma 3.2. Assume that $(\mathbb{L}, \mu)$ satisfies the UOSC. Then

$$
\#\left\{e \in \Xi_{\varepsilon}(\tau) \mid \overline{f_{e}(\mathcal{O})} \cap B(z, \varepsilon) \neq \varnothing\right\} \leq\left(4 / c_{\min }\right)^{d}
$$

for all $z \in F_{\tau}$ and $\varepsilon \in(0,1]$, where $\mathcal{O}$ is the open set guaranteed by the UOSC.

A proof for this lemma can be found in [Tro17a, Lemma 5.1.5] and [Tro19].

For ease of exposition we deal with the basic case where all maps in a fixed IFS contract equally. Recall that we assume $\mathbb{E}\left(\mathfrak{S}_{\omega_{1}}^{0}\right)>1$ throughout.

Theorem 3.3. Let $F_{\omega}$ be the random homogeneous attractor associated to the RIFS $(\mathbb{L}, \mu)$ satisfying the UOSC and suppose that $c_{\lambda}^{i}=c_{\lambda} \in\left[c_{\min }, c_{\max }\right]$ for every $i \in\left\{1, \ldots, \# \mathbb{I}_{\lambda}\right\}$ and $\lambda \in \Lambda$, where $0<c_{\min } \leq c_{\max }<1$. Let $\varepsilon>0, s=\operatorname{ess} \operatorname{dim}_{H} F_{\omega}$ and $\beta=\operatorname{Var}\left(\log \mathfrak{S}_{\omega_{1}}^{s}\right) / \eta$ for some $\eta \in \mathbb{R}$ (arising in the proof), then

$$
\mathscr{H}^{h_{1}(t, \beta, \varepsilon)}\left(F_{\omega}\right)=0,
$$

almost surely.

To prove this we need the Law of the iterated logarithm (LIL), see e.g. Athreya and Lahiri [AL06].

Proposition 3.4 (Law of the iterated logarithm (LIL)). Let $\left\{X_{i}\right\}_{i \in \mathbb{N}}$ be a sequence of i.i.d. random variables on a probability space $(\Omega, P)$ with mean $m_{0}=\int_{\Omega} X_{0} d P(\omega)$ and variance $V_{0}=\operatorname{Var}\left(X_{0}\right)$. Then, almost surely,

$$
\limsup _{k \rightarrow \infty} \frac{\sum_{i=1}^{k}\left(X_{i}-m_{0}\right)}{\sqrt{\left(2 V_{0} k \log \log V_{0} k\right)}}=1
$$

and similarly

$$
\limsup _{k \rightarrow \infty} \frac{\sum_{i=1}^{k}\left(X_{i}-m_{0}\right)}{\sqrt{\left(2 V_{0} k \log \log V_{0} k\right)}}=1 \text {. }
$$


Proof of Theorem 3.3. Let $\mathcal{O}$ be the open set guaranteed by the UOSC, we assume without loss of generality that $|\mathcal{O}|=1$. From the definition of Hausdorff measure

$$
\mathscr{H}^{h_{1}(t, \beta, \varepsilon)}\left(F_{\omega}\right) \leq \sum_{e \in \mathbf{C}_{\omega}^{k}} h_{1}\left(\left|\overline{f_{e}(\mathcal{O})}\right|, \beta, \varepsilon\right)
$$

for all $k \in \mathbb{N}$. So, writing $v=\operatorname{Var}\left(\log \mathfrak{S}_{\omega_{1}}^{s}\right)$,

$$
\begin{aligned}
& \mathscr{H}^{h_{1}(t, \beta, \varepsilon)}\left(F_{\omega}\right) \leq \liminf _{k \rightarrow \infty} \sum_{e \in \mathbf{C}_{\omega}^{k}} h_{1}\left(\left|\overline{f_{e}(\mathcal{O})}\right|, \beta, \varepsilon\right) \\
&= \liminf _{k \rightarrow \infty}\left(\prod_{i=1}^{k} \# \mathbb{I}_{\omega_{i}}\right)\left(c_{\omega_{1}} c_{\omega_{2}} \ldots c_{\omega_{k}}\right)^{s} \\
& \cdot \exp \left(\sqrt{2 \beta \log \left(1 /\left(c_{\omega_{1}} \ldots c_{\omega_{k}}\right)\right) \log \log \beta \log \left(1 /\left(c_{\omega_{1}} \ldots c_{\omega_{k}}\right)\right)}\right)^{1-\varepsilon} \\
&=\liminf _{k \rightarrow \infty} \exp \left[\left(\sum_{i=1}^{k} \log \mathfrak{S}_{\omega_{i}}^{s}\right)\right. \\
&\left.\quad+(1-\varepsilon) \sqrt{2 k \beta \log \left(C_{\omega}^{k}\right) \log \log \left(\beta k \log \left(C_{\omega}^{k}\right)\right)}\right]
\end{aligned}
$$

for $C_{\omega}^{k}=\left(c_{\omega_{1}} c_{\omega_{2}} \ldots c_{\omega_{k}}\right)^{-1 / k}$, and so

$$
\begin{aligned}
=\liminf _{k \rightarrow \infty} \exp \left[\left(\sum_{i=1}^{k} \log \mathfrak{S}_{\omega_{i}}^{s}\right)\right. & \\
& \left.+(1-\varepsilon) \sqrt{2 \frac{\log C_{\omega}^{k}}{\eta} k v \log \log \left(\frac{\log C_{\omega}^{k}}{\eta} k v\right)}\right] .
\end{aligned}
$$

Note that we can apply the law of the iterated logarithm, Proposition 3.4, to sums over the random variables $Y_{i}=\log \mathfrak{S}_{\omega_{i}}^{s}$ where $Y_{i}$ are i.i.d. with $\mathbb{E}\left(Y_{1}\right)=0$ and $0<\operatorname{Var}\left(Y_{1}\right)<\infty$. Thus

$$
\mathbb{P}_{H}\left\{\sum_{i=1}^{k} Y_{i} \leq-(1-\varepsilon / 2) \sqrt{2 v k \log \log (v k)} \text { for infinitely many } k \in \mathbb{N}\right\}=1
$$

Let $\left(i_{1}, i_{2}, \ldots\right)$ be a sequence of indices where the above inequality holds. Note that $c_{\min } \leq$ $C_{\omega}^{k}<c_{\max }$ for all $\omega$ and $k$, and so $\log c_{\min } \leq \log C_{\omega}^{k}<\log c_{\max }$. Therefore, for some uniform $\widetilde{\eta} \in\left[\log c_{\min }, \log c_{\max }\right]$, we have $\log C_{\omega}^{i_{k}} / \widetilde{\eta} \geq 1$ for infinitely many $k$, for almost all $\omega$. We can thus choose $\eta$ the greatest value for which this is satisfied.

We get, almost surely,

$$
\mathscr{H}^{h_{1}(t, \beta, \varepsilon)}\left(F_{\omega}\right) \leq \lim _{k \rightarrow \infty} \exp \left(-\frac{\varepsilon}{3} \sqrt{2 \operatorname{Var}\left(\log \mathfrak{S}_{\omega_{1}}^{s}\right) k \log \log \left(\operatorname{Var}\left(\log \mathfrak{S}_{\omega_{1}}^{s}\right) k\right)}\right)=0,
$$

completing the proof. 
We note that if $c_{\lambda}=\widetilde{c}$ for every $\lambda$, then $\eta=\log \widetilde{c}$. Note also the following corollary which implies that the 'fine dimension', i.e. the dimension according to the gauge function, is distinct from the random recursive case.

Corollary 3.5. Let $F_{\omega}$ be the random homogeneous attractor associated to the RIFS $(\mathbb{L}, \mu)$ satisfying the UOSC and suppose that $c_{\lambda}^{i}=c_{\lambda} \in\left[c_{\min }, c_{\max }\right]$ for every $i \in\left\{1, \ldots, \# \mathbb{I}_{\lambda}\right\}$ and $\lambda \in \Lambda$, where $0<c_{\min } \leq c_{\max }<1$. Let $h_{\beta}^{s}(t)=t^{s}(\log \log (1 / t))^{\beta}$, where $s=\operatorname{ess}_{\operatorname{dim}_{H}} F_{\omega}$ and $\beta>0$, then

$$
\mathscr{H}^{h_{\beta}^{s}(t)}\left(F_{\omega}\right)=0 . \quad \text { (a.s.) }
$$

Proof. We check

$$
\begin{aligned}
\lim _{t \rightarrow 0} \frac{h_{\beta}^{s}(t)}{h_{1}\left(t, \beta^{\prime}, \varepsilon\right)} & =\lim _{t \rightarrow 0} \frac{t^{s}(\log \log (1 / t))^{\beta}}{t^{s} \exp \left(\sqrt{2 \beta^{\prime}(\log (1 / t))\left(\log \log \log \left(1 / t^{\beta^{\prime}}\right)\right)}\right)^{1-\varepsilon}} \\
& =\lim _{t \rightarrow 0} \frac{(\log \log (1 / t))^{\beta}}{\exp \left((1-\varepsilon) \sqrt{2 \beta^{\prime}(\log (1 / t))\left(\log \log \log \left(1 / t^{\beta^{\prime}}\right)\right)}\right)} \\
& \leq \lim _{t \rightarrow 0} \frac{(\log (1 / t))^{\beta}}{\exp \left((1-\varepsilon) \sqrt{2 \beta^{\prime}(1 / t)}\right)}=0 .
\end{aligned}
$$

This holds for all $\beta, \beta^{\prime}, \varepsilon>0$ and the behaviour of the limits is sufficient for the desired result.

Considering $h_{1}(t, \beta,-\varepsilon)$ the law of the iterated logarithm guarantees a similar lower bound where the sum diverges. We will then use the mass distribution principle for the $h$-Hausdorff measure to establish infinite Hausdorff measure for $\varepsilon>0$.

Theorem 3.6 (Mass Distribution Principle). Let $\mu$ be a finite measure supported on $F$ and suppose that for some gauge function $h$ there are constants $c>0$ and $r_{0}$ such that $\mu(U) \leq c h(|U|)$ for all sets $U$ with $|U|<r_{0}$. Then $\mathscr{H}^{h}(F) \geq \mu(F) / c$.

While the proof can be found in a number of places (e.g. [Ols03]), we recall it for completeness.

Proof. Consider any countable open cover $\left\{O_{i}\right\}$ of $F$. Then

$$
\mu(F) \leq \mu\left(\bigcup_{i} O_{i}\right) \leq \sum_{i} \mu\left(O_{i}\right) \leq c \sum_{i} h\left(\left|O_{i}\right|\right)
$$

But then, taking the infimum for each $\delta>0$, we have $\mathscr{H}^{h}(F) \geq \mathscr{H}_{\delta}^{h}(F) \geq \mu(F) / c$.

Theorem 3.7. Let $F_{\omega}$ be the random homogeneous attractor associated to the RIFS $(\mathbb{L}, \mu)$ satisfying the UOSC and suppose that $c_{\lambda}^{i}=c_{\lambda} \in\left[c_{\min }, c_{\max }\right]$ for every $i \in\left\{1, \ldots, \# \mathbb{I}_{\lambda}\right\}$ and $\lambda \in \Lambda$, where $0<c_{\min } \leq c_{\max }<1$. Let $\varepsilon>0, s=\operatorname{ess~dim}_{H} F_{\omega}$ and $\beta_{0}=\eta_{0} \operatorname{Var}\left(\log \mathfrak{S}_{\omega_{1}}^{s}\right)$ for some $\eta_{0} \in \mathbb{R}$ (arising in the proof), then

$$
\mathscr{H}^{h_{1}\left(t, \beta_{0},-\varepsilon\right)}\left(F_{\omega}\right)=\infty
$$

holds almost surely. 
Proof. We use the same notation of the proof of Theorem 3.3. Let $\varepsilon>0$ be given and write $v=\operatorname{Var}\left(Y_{1}\right)$. Then the law of the iterated logarithm, Theorem 3.4, implies

$$
\mathbb{P}_{H}\left\{\sum_{i=1}^{k} Y_{i} \leq-(1+\varepsilon) \sqrt{2 v k \log \log (v k)} \text { for infinitely many } k \in \mathbb{N}\right\}=0
$$

and so, writing $D_{k}(\omega)=\left(c_{\omega_{1}} c_{\omega_{2}} \ldots c_{\omega_{k}}\right)^{-1}$, and $\eta_{k}(\omega)=k / \log D_{k}(\omega)$,

$$
\begin{aligned}
\mathbb{P}_{H}\left\{\mathfrak{S}_{\omega_{1}}^{s} \mathfrak{S}_{\omega_{2}}^{s} \ldots \mathfrak{S}_{\omega_{k}}^{s} \geq C \exp \left(-(1+\varepsilon) \sqrt{2 v \eta_{k}(\omega) \log \left(D_{k}(\omega)\right)}\right.\right. \\
\left.\left.\quad \cdot \sqrt{\log \log \left(v \eta_{k}(\omega) \log \left(D_{k}(\omega)\right)\right.}\right) \text { for all } k \geq l_{0}(\omega) \text { where } l_{0}(\omega) \in \mathbb{N}\right\}=1
\end{aligned}
$$

for any $C \in \mathbb{R}$. Since $c_{\lambda}$ is bounded away from 0 and 1 , the sequence $\eta_{k}(\omega)$ is uniformly bounded in $k$ and $\omega$. Therefore there exists uniform $\eta_{0}$ such that (3.1) holds with $\eta_{k}(\omega)$ replaced by $\eta_{0}$. Then, on a full measure set,

$$
\begin{aligned}
\left(\prod_{i=1}^{k} \mathcal{N}_{\omega_{i}}\right) & \geq C D_{k}(\omega)^{s} \exp \left(-(1+\varepsilon) \sqrt{2 v \eta_{0} \log \left(D_{k}(\omega)\right) \log \log \left(v \eta_{0} \log \left(D_{k}(\omega)\right)\right.}\right) \\
& =\frac{C}{h_{1}\left(D_{k}(\omega)^{-1}, \beta_{0},-\varepsilon\right)}
\end{aligned}
$$

holds for all $k \geq l_{0}(\omega)$.

We define a random measure $\nu_{\omega}$ on $F_{\omega}$. Assume $e \in \mathbf{C}_{\omega}^{k}$ for some $k \in \mathbb{N}$, for every basic cylinder we set

$$
\widetilde{\nu}_{\omega}\left(f_{e}(\mathcal{O})\right)=\left(\prod_{i=1}^{k} \mathcal{N}_{\omega_{i}}\right)^{-1}
$$

This extends to a unique random measure $\nu_{\omega}$ on $F_{\omega}$ for every $\omega \in \Omega$ by Carathéodory's extension theorem. We now show that, almost surely, there exists $C_{\omega}>0$ such that $\nu_{\omega}(U) \leq$ $\left(C_{\omega} / C\right) h_{1}\left(|U|, \beta_{0},-\varepsilon\right)$ for all small enough open $U$ that intersect $F_{\omega}$. Let $U$ be such that $u=2|U|<\left(c_{\min }\right)^{l_{0}(\omega)}$ and choose $z \in\left(U \cap F_{\omega}\right)$, then

$$
\begin{aligned}
& \nu_{\omega}(U) \leq \nu_{\omega}(B(z, u)) \leq \nu_{\omega}\left(\bigcup_{\substack{e \in \Xi_{u}(\omega) \\
f_{e}(\mathcal{O}) \cap B(z, u) \neq \varnothing}} f_{e}(\mathcal{O})\right) \\
& =\sum_{\substack{e \in \Xi_{u}(\omega) \\
f_{e}(\mathcal{O}) \cap B(z, u) \neq \varnothing}}\left(\prod_{i=1}^{k(u)} \mathcal{N}_{e_{i}}\right)^{-1} \leq\left(\frac{4}{c_{\min }}\right)^{d}\left(\prod_{i=1}^{k(u)} \mathcal{N}_{e_{i}}\right)^{-1}
\end{aligned}
$$

by Lemma 3.2, where $k(u)$ is the common length of all $e \in \Xi_{u}(\omega)$. Note that by assumption $k(u) \geq l_{0}(\omega)$. Therefore, using (3.2),

$$
\nu_{\omega}(U) \leq\left(\frac{4}{c_{\min }}\right)^{d} C^{-1} h_{1}\left(D_{k(u)}(\omega)^{-1}, \beta_{0},-\varepsilon\right) .
$$


Recall that $h_{1}$ is doubling, $c_{\omega_{1}} c_{\omega_{2}} \ldots c_{\omega_{k}}=D_{k}(\omega)<u$, and so there exists $\kappa>0$ such that

$$
\nu_{\omega}(U) \leq(\kappa / C) h_{1}\left(|U|, \beta_{0},-\varepsilon\right) .
$$

Now, using the mass distribution principle, Theorem 3.6, we conclude

$$
\mathscr{H}^{h_{1}\left(t, \beta_{0},-\varepsilon\right)}\left(F_{\omega}\right) \geq \frac{C}{\kappa} .
$$

The desired conclusion follows from the fact that $C$ was arbitrary.

Note that the constants $\eta$ and $\eta_{0}$ might not coincide, and thus $\beta=\beta_{0}$ might not hold.

Both Theorems 3.3 and 3.7 seems to suggest that $h_{1}(t, \beta, 0)$ with $\beta=\beta_{0}$ is the correct function that gives positive and finite Hausdorff measure. However, we will now show that not only is there no $\beta$ such that

$$
0<\mathscr{H}^{h_{1}(t, \beta, 0)}\left(F_{\omega}\right)<\infty
$$

but there does not exist any gauge function $h$ that gives positive and finite Hausdorff measure.

\section{The non-existence of gauge functions for self-similar code-trees with necks}

In this section we will prove our main result, namely that random code-trees with necks and random homogeneous attractors do not admit a gauge function, provided they are not almost deterministic. First, we will prove the equicontractive random homogeneous case as its proof is considerably simpler than the full case. Further, the simplistic equicontractive case gives a better intuition as to why the gauge function cannot exist, something that can more easily get lost in full generality.

Before we deal with these we first show that we can without loss of generalisation assume $h(t)$ is of the form $h(t)=t^{s+g(t)}$ where $g(t) \nearrow 0$ from below as $t \rightarrow 0$. Consider a self-similar code-tree with necks. By Condition 2.2 the attractor has positive Hausdorff dimension. Since having Hausdorff dimension greater than $\alpha>0$ is a tail event, ergodicity implies that there exists an almost sure Hausdorff dimension $s$. Thus $\log (h(t)) / \log (t) \rightarrow s$ and $g(t) \rightarrow 0$. Further, $\mathscr{H}^{s}\left(F_{\tau}\right)=0$ almost surely and we must have $g(t) \leq 0$ for positive $h$-Hausdorff measure. Lastly, we can assume $g(t)$ to be increasing as there is no 'preferred' scale, i.e. the contractions that are randomly chosen are bounded away from 0 and 1 and the expected block size is finite, and having positive and finite measure is a tail event with probability 0 or 1.

\subsection{Equicontractive homogeneous random attractors}

Let us first consider the case when $(\mathbb{L}, \mu)$ satisfies the UOSC and all maps in all IFSs have the same contraction rate $c \in(0,1)$. Then,

$$
\sum_{e \in \mathbf{C}_{\omega}^{k}} h(c)=\left(\prod_{i=1}^{k} \mathcal{N}_{\omega_{i}}\right) h\left(c^{k}\right) .
$$


As we will show below, finding a gauge function for positive and finite Hausdorff measure reduces to the problem of finding a gauge function such that

$$
\liminf _{k \rightarrow \infty}\left(\prod_{i=1}^{k} \mathcal{N}_{\omega_{i}}\right) h\left(c^{k}\right) \in(0, \infty)
$$

almost surely. But, for $h_{k}=\log h\left(c^{k}\right)$,

$$
\liminf _{k \rightarrow \infty}\left(\prod_{i=1}^{k} \mathcal{N}_{\omega_{k}}\right) h\left(c^{k}\right)=\liminf _{k \rightarrow \infty} \exp \left(h_{k}+\sum_{i=1}^{k} \log \mathcal{N}_{\omega_{i}}\right) .
$$

Therefore, the problem of finding a suitable gauge function becomes equivalent to finding a (fixed) sequence $\left(h_{k}\right)$ such that $\liminf _{k \rightarrow \infty} h_{k}+\sum_{i=1}^{k} X_{i}$ is positive and finite for some sequence of i.i.d. random variables $X_{i}$ with positive variance. Something that clearly does not exist by the Central Limit Theorem.

Since the general case is somewhat more complex to deal with, we will first prove the same result for equicontractive sets using methods that will generalise more readily. Throughout the remainder of this section we assume that $(\mathbb{L}, \mu)$ satisfies the UOSC and is equicontractive, i.e. for every $\lambda \in \Lambda$ all maps $f_{\lambda}^{i} \in \mathbb{I}_{\lambda}$ have contraction ratio $c_{\lambda}^{i}=c_{\lambda}$. This is slightly more general than the case considered just above. We will continue to assume Condition 2.2 and that $(\mathbb{L}, \mu)$ is not almost deterministic. In particular, this means that there exists $0<\gamma<1$ and $\varepsilon>0$ such that

$$
p_{0}:=\mu\left\{\sum_{f_{\lambda}^{i} \in \mathbb{I}_{\lambda}}\left(c_{\lambda}\right)^{s-\varepsilon} \leq \gamma\right\}>0
$$

where $s$ is the almost sure Hausdorff dimension of the homogeneous random attractor $F_{\omega}$ with $\omega \in \Omega=\Lambda^{\mathbb{N}}$ and $\mathbb{P}_{H}=\mu^{\mathbb{N}}$.

Let $h$ be an arbitrary gauge function. Given $\omega \in \Omega$ we obtain

$$
\sum_{e \in \mathbf{C}_{\omega}^{k}} h\left(c_{e}\right)=\sum_{e \in \mathbf{C}_{\omega}^{k}} h\left(c_{\omega_{1}} c_{\omega_{2}} \ldots c_{\omega_{k}}\right)=\left(\prod_{i=1}^{k} \mathcal{N}_{\omega_{i}}\right) h\left(c_{\omega_{1}} c_{\omega_{2}} \ldots c_{\omega_{k}}\right) .
$$

The equicontractive case is simpler for two reasons. First, it allows us to write the sum of gauge values as the product of the number of descendants with the gauge function at a single value. Further, the equicontractive property also implies that the Hausdorff measure is given by the lower limit of the sums of gauge functions values.

Lemma 4.1. Let $(\mathbb{L}, \mu)$ be a RIFS that satisfies the UOSC, Condition 2.2, is equicontractive, and let $F_{\omega}$ be the associated random homogeneous attractor. Then, for any gauge function $h$, and all $\omega \in \Omega$ there exists $\kappa_{\omega}>0$ such that

$$
\mathscr{H}^{h}\left(F_{\omega}\right)=\kappa_{\omega} \liminf _{k \rightarrow \infty} \sum_{e \in \mathbf{C}_{\omega}^{k}} h\left(c_{e}\right) .
$$

Proof. Let $\mathcal{O}$ be the open set guaranteed by the UOSC. Clearly,

$$
F_{\omega} \subseteq \bigcup_{e \in \mathbf{C}_{\omega}^{k}} \overline{f_{e}(\mathcal{O})}
$$

and so

$$
\mathscr{H}^{h}\left(F_{\omega}\right) \leq \kappa_{\omega} \liminf _{k \rightarrow \infty} \sum_{e \in \mathbf{C}_{\omega}^{k}} h\left(c_{e}\right)
$$


for some $\kappa_{\omega}$. For the lower bound, we see that $\left\{\overline{f_{e}(\mathcal{O})}\right\}_{e \in \mathbf{C}_{\omega}^{*}}$ is a Vitali cover. A standard argument then gives that for every $\varepsilon>0$ there exist subsets of $C_{k} \subset \mathbf{C}_{\omega}^{*}$ such that for every $v, w \in C_{k}$, neither $v$ is a parent of $w$ or vice versa, and that every $v \in C_{k}$ has length at least $k$. Using Lemma 3.2 , we can extend $C_{k}$ to a full tree by admitting at most $\left(4 / c_{\min }\right)^{d}$ many further cylinders. Thus, for some set $C_{k}^{\prime}$,

$$
\frac{1}{\left(4 / c_{\min }\right)^{d}} \sum_{e \in C_{k}^{\prime}} h\left(c_{e} \cdot|\mathcal{O}|\right) \leq \mathscr{H}^{h}\left(F_{\omega}\right)+\varepsilon .
$$

Now note that by homogeneity, we can assume that all $e \in C_{k}^{\prime}$ must be at the same tree level $k$. This follows from the observation that, if $e$ and $f$ are two different nodes in levels $k_{e}>k_{f}$ then there exists a parent of $e$, say $e^{\prime}$, in level $k_{f}$ that does not have $f$ as a descendant. Further, there exists a (non-trivial) collection of descendants $e_{i}$ such that $\sum h\left(c_{e_{i}}\right) \sim h\left(c_{f}\right)$. But since all the descendants of $f$ behave exactly as the descendants of $e^{\prime}$, we do not need to consider the children of $e^{\prime}$ for an efficient cover. By induction this extends to a comparable cover over a single tree level and

$$
\kappa_{\omega} \liminf _{k \rightarrow \infty} \sum_{e \in \mathbf{C}_{\omega}^{k}} h\left(c_{e}\right) \leq \mathscr{H}^{h}\left(F_{\omega}\right)+\varepsilon
$$

for some $\kappa_{\omega}>0$. Since $\varepsilon$ was arbitrary, the desired conclusion follows.

We are now ready to state and prove our main theorem of this section.

Theorem 4.2. Let $(\mathbb{L}, \mu)$ be a RIFS that satisfies the UOSC, Condition 2.2, is equicontractive, and let $F_{\omega}$ be the associated random homogeneous attractor. Let $h$ be a gauge function, then for almost all $\omega \in \Omega$,

$$
\mathscr{H}^{h}\left(F_{\omega}\right) \in\{0, \infty\} .
$$

In particular, there exists no gauge function such that the $h$-Hausdorff measure is positive and finite almost surely.

Proof. In light of Lemma 4.1 all that remains is to show that the right hand side of (4.2) is 0 or $\infty$ almost surely, irrespective of $h$. Now $\left\{0<\mathscr{H}^{h}\left(F_{\omega}\right)<\infty\right\}$ is a tail-event and thus has probability 0 or 1 by Kolmogorov's Zero - One Law. Therefore, we assume for a contradiction that

$$
\mathbb{P}_{H}\left\{\omega \in \Omega \mid \liminf _{k \rightarrow \infty} \sum_{e \in \mathbf{C}_{\omega}^{k}} h\left(c_{e}\right) \in(0, \infty)\right\}=1 .
$$

Recall equation (4.1), which implies that there exists positive probability $p_{0}$ such that a letter $\lambda$ is picked that lowers the sum. Call the set of letters $B$. Using the assumption that $h(t)=t^{s+g(t)}$ for some decreasing $g(t) \leq 0$, let $\varepsilon>0$ be as given in (4.1) and choose $t_{0}$ such that $g(t) \geq-\varepsilon$ for all $0 \leq t<t_{0}$. Then,

$$
\begin{aligned}
\sup _{\lambda \in B} \sum_{f_{\lambda}^{i} \in \mathbb{I}_{\lambda}} h\left(c_{\lambda} \cdot t\right) & =\sup _{\lambda \in B} \sum_{f_{\lambda}^{i} \in \mathbb{I}_{\lambda}}\left(c_{\lambda} \cdot t\right)^{s+g\left(c_{\lambda} \cdot t\right)} \\
& \leq \sup _{\lambda \in B} \sum_{f_{\lambda}^{i} \in \mathbb{I}_{\lambda}}\left(c_{\lambda} \cdot t\right)^{s+g(t)} \\
& \leq h(t) \sup _{\lambda \in B} \sum_{f_{\lambda}^{i} \in \mathbb{I}_{\lambda}}\left(c_{\lambda}\right)^{s-\varepsilon} \leq \gamma h(t)
\end{aligned}
$$


for some $0<\gamma<1$ as given in (4.1).

For $i \in \mathbb{Z}$, we define level sets $E_{i}$ by

$$
E_{i}:=\left\{\omega \in \Omega \mid \liminf _{k \rightarrow \infty} \sum_{e \in \mathbf{C}_{\omega}^{k}} h\left(c_{e}\right) \in\left(\gamma^{i+1}, \gamma^{i}\right]\right\}
$$

Clearly, $\bigcup_{i \in \mathbb{Z}} E_{i}$ is a disjoint union with full measure and there exists $j_{0} \in \mathbb{Z}$ such that $\mathbb{P}_{H}\left(E_{j_{0}}\right)>0$. Since $c_{\max }<1$, there exists $k_{0}$ such that $c_{e}<t_{0}$ for all $e \in \mathbf{C}_{\omega}^{k}$, where $k>k_{0}$. For $\omega \in \bigcup_{i \in \mathbb{Z}} E_{i}$ we write $\zeta_{k}(\omega)$ for the $k$-th time of being close to the lower limit, i.e.

$$
\zeta_{1}(\omega)=\min \left\{k>k_{0} \mid \sum_{e \in \mathbf{C}_{\omega}^{k}} h\left(c_{e}\right) \leq \gamma^{i} \text { and } \sum_{e \in \mathbf{C}_{\omega}^{l}} h\left(c_{e}\right)>\gamma^{i+1},(\forall l \geq k) \text {, where } \omega \in E_{i}\right\}
$$

and

$$
\begin{aligned}
\zeta_{j}(\omega)=\min \left\{k>\zeta_{j-1}(\omega) \mid\right. & \sum_{e \in \mathbf{C}_{\omega}^{k}} h\left(c_{e}\right) \leq \gamma^{i} \text { and } \\
& \left.\sum_{e \in \mathbf{C}_{\omega}^{l}} h\left(c_{e}\right)>\gamma^{i+1},(\forall l \geq k), \text { where } \omega \in E_{i}\right\}
\end{aligned}
$$

Recall that positive and finite Hausdorff measure is a tail event. Thus, inserting a letter almost never changes the Hausdorff measure from a positive and finite value to one in $\{0, \infty\}$. We therefore assume, without loss of generality, that the insertion of a letter does not change the word belonging to the tail event $\left\{0<\mathscr{H}^{h}\left(F_{\omega}\right)<\infty\right\}$.

Now consider $\widehat{E}_{j_{0}}(i)$, where

$$
\begin{aligned}
& \widehat{E}_{j_{0}}(i)=\left\{\omega \in \Omega \mid \exists \omega^{\prime} \in E_{j_{0}} \text { such that } \omega_{k}=\omega_{k}^{\prime} \text { for } 1 \leq k \leq \zeta_{j}\left(\omega^{\prime}\right),\right. \\
& \text { and } \left.\omega_{\zeta_{j}\left(\omega^{\prime}\right)+1} \in B \text {, and } \omega_{k+1}=\omega_{k}^{\prime} \text { for } k>\zeta_{j}\left(\omega^{\prime}\right)\right\} .
\end{aligned}
$$

It is immediate that $\mathbb{P}_{H}\left(\widehat{E}_{j_{0}}(i)\right)=p_{0} \mathbb{P}_{H}\left(E_{j_{0}}\right)>0$ and $\widehat{E}_{j_{0}}(i) \subset \bigcup_{k<j_{0}} E_{k}$. Further, for every $\omega \in E_{i}$, the letters at positions $\zeta_{j}(\omega)$ cannot be followed by a letter $b \in B$. This is because if such a letter was at this position, by equation (4.3), $\omega \in E_{l}$ for some $l<i$, a contradiction to the initial hypothesis. Since $\omega_{\zeta_{j}(\omega)+1} \notin B$ we must also have $E_{j_{0}}(i) \cap$ $E_{j_{0}}\left(i^{\prime}\right)=\varnothing$ for all $i \neq i^{\prime}$, as otherwise it would contradict the ordering of exceptional positions. But then

$$
1=\mathbb{P}_{H}\left(\bigcup_{i<j_{0}} E_{i}\right) \geq \mathbb{P}_{H}\left(\bigcup_{i \in \mathbb{N}} \widehat{E}_{j_{0}}(i)\right)=\sum_{i \in \mathbb{N}} \mathbb{P}_{H}\left(\widehat{E}_{j_{0}}(i)\right)=\sum_{i \in \mathbb{N}} p_{0} \mathbb{P}_{H}\left(E_{j_{0}}\right)=\infty,
$$

a contradiction. Therefore

$$
\mathbb{P}_{H}\left\{\omega \in \Omega \mid \liminf _{k \rightarrow \infty} \sum_{e \in \mathbf{C}_{\omega}^{k}} h\left(c_{e}\right) \in(0, \infty)\right\}=0,
$$

proving our main statement. 


\subsection{Gauge functions for random code-trees with necks}

The proof for equicontractive systems is fairly straightforward and its generalisation above relied to a heavy degree on the comparability of the evaluation over level sets with the Hausdorff measure. Allowing different contraction rates, this no longer holds for all $\omega$. Our proof for the general case will imply that they are still comparable almost surely, but we do not know this a priori. Instead, we will consider splitting up the tree and assigning values to each node corresponding to the minima achieved below it. We will then adopt the method above to obtain a similar contradiction to the positivity and finiteness assumption.

We start by extending the almost deterministic condition to random code-trees with necks.

Definition 4.3. Let $\mathbb{L}$ be a family of IFSs that satisfy the UOSC and Condition 2.2. Further, let $\mathbb{P}$ be a code-tree measure (see Definition 1.7). We call the random code-tree fractal associated with $\mathbb{P}$ almost deterministic if

$$
\mathbb{P}\left\{\tau \in \mathcal{T} \mid \sum_{e \in \mathbf{T}_{\tau}^{N_{1}(\tau)}}\left(c_{e}\right)^{s}=1\right\}=1,
$$

where $s$ is the almost sure Hausdorff dimension of $F_{\tau}$.

Since $N_{1}(\tau)=1$ for all random homogeneous attractors, these definitions coincide. Note, as in the equicontractive random homogeneous case, that if the random code-tree fractal is not almost deterministic, there exist $0<p_{0}, \varepsilon, \gamma<1$ such that

$$
\mathbb{P}\left\{\tau \in \mathcal{T} \mid \sum_{e \in \mathbf{T}_{\tau}^{N_{1}(\tau)}}\left(c_{e}\right)^{s-\varepsilon} \leq \gamma\right\}=p_{0}
$$

where $s$ is again the almost sure Hausdorff dimension of $F_{\tau}$. This allows us to establish a similar drop in the gauge function.

Lemma 4.4. Let $\mathbb{L}$ be a family of IFS that satisfy the UOSC and Condition 2.2. Let $\mathbb{P}$ be a random code-tree measure and assume that $F_{\tau}$ is not almost deterministic. Further, let $h(t)$ be any gauge function. Then there exist $B \subset \mathcal{T}$ and $t_{0} \in(0,1)$ such that $\mathbb{P}(B)=p_{0}>0$ and for all $0<t \leq t_{0}$,

$$
\sup _{\tau \in B} \sum_{e \in \mathbf{T}_{\tau}^{N_{1}(\tau)}} h\left(t \cdot c_{e}\right) \leq \gamma h(t)
$$

The proof is almost identical to the first part of the proof of Theorem 4.2 and omitted here. If we were able to prove that

$$
\mathscr{H}^{h}\left(F_{\tau}\right) \asymp \liminf _{k \rightarrow \infty} \sum_{e \in \mathbf{T}_{\tau}^{N_{k}(\tau)}} h\left(c_{e}\right),
$$

we could use the same strategy as in Theorem 4.2. However, it seems unlikely to hold in generality as we have no control over the behaviour in between neck levels. We shall use a different, yet in same ways similar strategy to prove our main theorem.

Theorem 4.5 (Main Theorem). Let $\mathbb{L}$ be a family of IFS that satisfy the UOSC and Condition 2.2. Let $\mathbb{P}$ be a random code-tree measure and assume that $F_{\tau}$ is not almost deterministic. Further, let $h(t)$ be any gauge function. Then,

$$
\mathbb{P}\left\{\tau \in \mathcal{T} \mid \mathscr{H}^{h}\left(F_{\tau}\right) \in\{0, \infty\}\right\}=1 .
$$

In particular, there does not exist a gauge function that gives positive and finite measure almost surely. 
Before we give the proof we recall the notion of sections and minimal sections.

Definition 4.6. Let $\Sigma^{*}$ be the $\mathcal{N}$-ary tree. A finite subset $M \subset \Sigma^{*}$ is called a section if every long enough node $v \in \Sigma^{*}$ has an initial word in $M$. That is, there exists some $l_{0}$ such that for every $l \geq l_{0}$ and every $v \in \Sigma_{l}$ there exists $w \in M$ of length $l_{w}$ such that $v_{i}=w_{i}$ for all $1 \leq i \leq l_{w}$. A section $M$ is referred to as a minimal section if no proper subset of $M$ is a section.

Proof of Theorem 4.5. Recall that $\mathcal{O}$ is the set guaranteed by the UOSC. We start by assuming, for a contradiction, that with positive probability $0<\mathscr{H}^{h}\left(F_{\tau}\right)<\infty$. Since we are again dealing with a tail event, we can further assume that $\mathbb{P}\left\{0<\mathscr{H}^{h}\left(F_{\tau}\right)<\infty\right\}=1$ by Kolmogorov's Zero - One Law. Instead of relating the Hausdorff measure to level sets, we will relate them to tree sections.

For any section $M \subset \Sigma^{*}$,

$$
F_{\tau} \subseteq \bigcup_{v \in M} \overline{f_{\mathbf{T}_{\tau}(v)}(\mathcal{O})}
$$

and so

$$
\mathscr{H}^{h}\left(F_{\tau}\right) \leq \sum_{v \in M} h\left(\overline{f_{\mathbf{T}_{\tau}(v)}(\mathcal{O})} \mid\right)=\sum_{v \in M} h\left(c_{\mathbf{T}_{\tau}(v)} \cdot|\mathcal{O}|\right) \leq \kappa_{\tau} \sum_{v \in M} h\left(c_{\mathbf{T}_{\tau}(v)}\right),
$$

for some $\kappa_{\tau}>0$. In particular this holds for all minimal sections, and we will use these to obtain a lower bound to the Hausdorff measure. Let $M_{k}$ be a minimal section such that every $v \in M_{k}$ has length at least $k$ and let $\mathbf{M}_{k}$ be the set of all such minimal sections. Assuming the uniform strong separation condition, i.e. $f_{\lambda_{1}}(\Delta) \cap f_{\lambda_{2}}(\Delta) \neq \varnothing$ implies $\lambda_{1}=\lambda_{2}$, where $\lambda_{1}, \lambda_{2} \in \Lambda$ and $\Delta$ is as in Definition 1.2, it is easy to show that

$$
\mathscr{H}^{h}\left(F_{\tau}\right)=\lim _{k \rightarrow \infty} \inf _{M_{k} \in \mathbf{M}_{k}} \sum_{v \in M} h\left(c_{\mathbf{T}_{\tau}(v)} \cdot\left|F_{\tau}\right|\right) .
$$

We refer the reader to Furstenberg [Fur14] which provides a proof for $h(t)=t^{s}$. The general gauge function case is identical and left to the reader. First, notice that by similar reasons to Theorem 4.2 we can, without loss of generality, assume

$$
\lim _{k \rightarrow \infty} \inf _{M_{k} \in \mathbf{M}_{k}} \sum_{v \in M} h\left(c_{\mathbf{T}_{\tau}(v)} \cdot\left|F_{\tau}\right|\right) \asymp \lim _{k \rightarrow \infty} \inf _{M_{k} \in \mathbf{M}_{k}} \sum_{v \in M} h\left(c_{\mathbf{T}_{\tau}(v)}\right) .
$$

However, we are interested in the case where $\mathbb{L}$ satisfies the UOSC rather than the uniform strong separation condition. To this end, we can modify our sections to only allow elements that do not overlap, thus artificially forcing strong separation. That is,

$$
\mathscr{H}^{h}\left(F_{\tau}\right) \geq \kappa_{\tau} \lim _{k \rightarrow \infty} \inf _{M_{k} \in \mathbf{M}_{k}}\left\{\operatorname { s u p } _ { \widehat { M } \subseteq M _ { k } } \left\{\sum_{v \in \widehat{M}} h\left(c_{\mathbf{T}_{\tau}(v)}\right) \mid \overline{f_{\mathbf{T}_{\tau}(v)}(\mathcal{O})} \cap \overline{f_{\mathbf{T}_{\tau}(w)}(\mathcal{O})}=\varnothing\right.\right.
$$

for distinct $v, w \in \widehat{M}\}\}$.

for some $\kappa_{\tau}$. But, using Lemma 3.2, 


$$
\begin{aligned}
& \lim _{k \rightarrow \infty} \inf _{M_{k} \in \mathbf{M}_{k}}\left\{\operatorname { s u p } _ { \widehat { M } \subseteq M _ { k } } \left\{\sum_{v \in \widehat{M}} h\left(c_{\mathbf{T}_{\tau}(v)}\right) \mid \overline{f_{\mathbf{T}_{\tau}(v)}(\mathcal{O})}\right.\right. \cap \overline{f_{\mathbf{T}_{\tau}(w)}(\mathcal{O})}=\varnothing \\
&\quad \text { for distinct } v, w \in \widehat{M}\}\} \\
& \geq\left(\frac{c_{\min }}{4}\right)^{d} \lim _{k \rightarrow \infty} \inf _{M_{k} \in \mathbf{M}_{k}} \sum_{v \in M} h\left(c_{\mathbf{T}_{\tau}(v)}\right) .
\end{aligned}
$$

We conclude,

$$
\mathscr{H}^{h}\left(F_{\tau}\right) \in(0, \infty) \quad \text { if and only if } \quad \lim _{k \rightarrow \infty} \inf _{M_{k} \in \mathbf{M}_{k}} \sum_{v \in M} h\left(c_{\mathbf{T}_{\tau}(v)}\right) \in(0, \infty) .
$$

and redefine $\kappa_{\tau}$ such that

$$
\kappa_{\tau} \cdot \mathscr{H}^{h}\left(F_{\tau}\right)=\lim _{k \rightarrow \infty} \inf _{M_{k} \in \mathbf{M}_{k}} \sum_{v \in M} h\left(c_{\mathbf{T}_{\tau}(v)}\right),
$$

setting $\kappa_{\tau}=1$ for all $\tau$ such that $\mathscr{H}^{h}\left(F_{\tau}\right) \in\{0, \infty\}$.

Instead of tree levels, we consider minimal subsections at neck levels. Let $\mathbf{M}^{*}=\bigcup M_{k}$ and set

$$
S_{\tau}(t)=\inf _{M_{k} \in \mathbf{M}^{*}} \sum_{v \in M} h\left(t \cdot c_{\mathbf{T}_{\tau}(v)}\right) .
$$

Clearly,

$$
\begin{aligned}
\lim _{k \rightarrow \infty} \sum_{v \in \Sigma_{k}} S_{\sigma^{v} \tau}\left(\mathbf{T}_{\tau}(v)\right) & =\lim _{k \rightarrow \infty} \sum_{v \in \Sigma_{N_{k}(\tau)}} S_{\sigma^{v} \tau}\left(\mathbf{T}_{\tau}(v)\right) \\
& =\lim _{k \rightarrow \infty} \inf _{M_{k} \in \mathbf{M}_{k}} \sum_{v \in M} h\left(c_{\mathbf{T}_{\tau}(v)}\right)=\kappa_{\tau} \mathscr{H}^{h}\left(F_{\tau}\right),
\end{aligned}
$$

as the leftmost sum is non-decreasing in $k$, for all $\tau \in \mathcal{T}$. So, our initial assumption is also equivalent to $\mathbf{S}_{\tau}^{k}=\sum_{v \in \Sigma_{k}} S_{\sigma^{v} \tau}\left(\mathbf{T}_{\tau}(v)\right)$ converging to some non-zero and finite value from below for almost every $\tau$.

Let $\mathcal{T}^{\prime}$ denote the full measure set for which there exist infinitely many necks. We define

$$
E=\left\{\tau \in \mathcal{T}^{\prime} \mid \mathbf{S}_{\tau}^{k}<\kappa_{\tau} \mathscr{H}^{h}\left(F_{\tau}\right) \text { for all } k\right\}
$$

to be the set of realisations that converge properly to its limit. First, we assume that $\mathbb{P}(E)>0$ to derive a contradiction in a similar spirit to the homogeneous case. Let $k_{0}$ be such that $c_{\mathbf{T}_{\tau}(v)}<t_{0}$ for all $v \in \bigcup_{k \geq k_{0}} \Sigma_{k}$. For $i \in \mathbb{Z}$, we analogously define level sets $E_{i}$ by

$$
E_{i}:=\left\{\tau \in \mathcal{T}^{\prime} \mid \lim _{k \rightarrow \infty} \mathbf{S}_{\tau}^{k} \in\left(\gamma^{i+1}, \gamma^{i}\right]\right\}
$$

where $\gamma$ is given in Lemma 4.4. Again, $E^{*}=\bigcup_{i \in \mathbb{Z}} E_{i}$ is a disjoint union with full measure and so $\mathbb{P}\left(E^{*} \cap E\right)>0$. Thus there exists $j_{0} \in \mathbb{Z}$ such that $\mathbb{P}\left(E \cap E_{j_{0}}\right)>0$.

For $\tau \in E^{*}$ we write $\zeta_{k}(\tau)$ for the $k$-th neck level not less than $k_{0}$ such that a 'jump' in value occurs at the next neck. i.e.

$$
\zeta_{1}(\tau)=\min \left\{k>k_{0} \mid \mathbf{S}_{\tau}^{N_{k}(\tau)}<\mathbf{S}_{\tau}^{N_{k+1}(\tau)}\right\}
$$


and

$$
\zeta_{j}(\tau)=\min \left\{k>\zeta_{j-1}(\omega) \mid \mathbf{S}_{\tau}^{N_{k}(\tau)}<\mathbf{S}_{\tau}^{N_{k+1}(\tau)}\right\}
$$

First note that for a given $\tau \in E^{*}$, there must be some $v \in \Sigma^{*}$ of length $N_{k}(\tau) \leq|v|<$ $N_{k+1}(\tau)$ such that $S_{\sigma^{v} \tau}\left(\mathbf{T}_{\tau}(v)\right)=h\left(\mathbf{T}_{\tau}(v)\right)$ as otherwise the value would not jump. Further, this jump implies that the shift $\sigma^{v_{k}} \tau \notin B$ for $v_{k}=(1,1, \ldots, 1) \in \Sigma_{N_{k}}$. Thus we can form a new set by inserting a new neck block at this jump value that decreases the Hausdorff measure and set

$$
\begin{array}{r}
\widehat{E}_{j_{0}}(i)=\left\{\tau \in \mathcal{T}^{\prime} \mid \exists b \in B, \exists \tau^{\prime} \in E_{j_{0}} \text { such that } \tau(v)=\tau^{\prime}(v) \text { for all } v \in \bigcup_{k=1}^{N_{\zeta_{i}}\left(\tau^{\prime}\right)-1} \Sigma_{k},\right. \\
\text { and } \sigma^{v} \tau=b \text { for all } v \in \Sigma_{N_{\zeta_{i}}\left(\tau^{\prime}\right)} \text { such that } \mathbf{T}(v) \neq \emptyset \text {, and } \sigma^{v} \tau=\sigma^{v_{k+1}} \tau^{\prime} \\
\text { for all } \left.v \in \Sigma_{N_{\zeta_{i}}\left(\tau^{\prime}\right)+N_{1}(b)} \text { such that } \mathbf{T}(v) \neq \emptyset\right\} .
\end{array}
$$

Now $\mathbb{P}$ is invariant with respect to neck-shifts and in-between necks are independent. We can therefore conclude that $\mathbb{P}\left(\widehat{E}_{j_{0}}(i)\right)=p_{0} \mathbb{P}\left(E_{j_{0}}\right)>0$ and obtain

$$
1=\mathbb{P}\left(\bigcup_{i<j_{0}} E_{i}\right) \geq \mathbb{P}\left(\bigcup_{i \in \mathbb{N}} \widehat{E}_{j_{0}}(i)\right)=\sum_{i \in \mathbb{N}} \mathbb{P}\left(\widehat{E}_{j_{0}}(i)\right)=\sum_{i \in \mathbb{N}} p_{0} \mathbb{P}\left(E_{j_{0}}\right)=\infty,
$$

and so $\mathbb{P}(E)=0$.

Since we have reached a contradiction, we must conclude that

$$
K=\left\{\tau \in \mathcal{T}^{\prime} \mid \exists k_{1}(\tau)>k_{0} \text { such that } \mathbf{S}_{\tau}^{k}=\kappa_{\tau} \mathscr{H}^{h}\left(F_{\tau}\right) \text { for all } k \geq k_{1}(\tau)\right\}
$$

has full measure. We immediately conclude that sums of any section with word length at least $k_{1}(\tau)$ give an upper bound. Since neck levels are independent we can also conclude that $K \cap B \cap \mathcal{T}^{\prime}$ has full measure.

Let $S$ be all minimal sections such that all $v \in S$ have length greater than $k_{1}(\tau)$ and

$$
\kappa_{\tau} \mathscr{H}^{h}\left(F_{\tau}\right) \leq \sum_{v \in S} h\left(\mathbf{T}_{\tau}(v)\right)<\frac{\kappa_{\tau}}{\gamma} \mathscr{H}^{h}\left(F_{\tau}\right) .
$$

There must exist $v_{0} \in S$ such that $S_{\sigma^{v_{0}}}\left(\mathbf{T}_{\tau}\left(v_{0}\right)\right) \leq h\left(\mathbf{T}_{\tau}\left(v_{0}\right)\right)<\gamma^{-1} S_{\sigma^{v_{0}}}\left(\mathbf{T}_{\tau}\left(v_{0}\right)\right)$. Let $l_{0}$ be the length of $v_{0}$. If $l_{0}=N_{k}(\tau)$ for some $k$, then the next neck cannot lie in $B$. Similarly, if $N_{k}(\tau)<l_{0} \leq N_{k+1}(\tau)$ we can use the maximal contraction rate $c_{\max }$ and the maximal splitting $\mathcal{N}$ to conclude that there cannot be more than $n_{0}=\left(N_{k+1}-N_{k}\right) \log \left(\mathcal{N}\left(c_{\max }\right)^{s-\varepsilon}\right) / \log \gamma$ consecutive necks in $B$. However, under our assumptions, the value of $S_{\sigma^{v}}\left(\mathbf{T}_{\tau}\left(v_{0}\right)\right)$ does not depend on the order of the letters past $l_{0}$ and there cannot be more than $n_{0}$ occurrences of necks in $B$ after $k_{1}(\tau)$. Hence there are only finitely many necks shifts in $B$, a contradiction. We conclude that, almost surely, the $h$-Hausdorff measure is zero or infinite. 


\section{$5 \quad$ Exact packing measure}

The packing measure can be considered the dual of the Hausdorff measure. For arbitrary gauge functions we define it thus.

Definition 5.1. Let $F \subseteq \mathbb{R}^{d}$ and $h(t)$ be a gauge function. Define

$$
\begin{aligned}
& \mathscr{P}_{\delta}^{h}(F)=\sup \left\{\sum_{i} h\left(\left|B_{i}\right|\right) \mid\left\{B_{i}\right\}\right. \text { is a countable collection of disjoint balls } \\
& \text { centred in } \left.F \text { with radii } r_{i} \leq \delta\right\}
\end{aligned}
$$

and set $\mathscr{P}_{0}^{h}(F)=\lim _{\delta \rightarrow \infty} \mathscr{P}_{\delta}^{h}(F)$. The packing measure is

$$
\mathscr{P}^{h}(F)=\inf \left\{\sum_{i=1}^{\infty} \mathscr{P}_{0}^{s}\left(F_{i}\right) \mid \text { where } f \subseteq \bigcup_{i=1}^{\infty} F_{i}\right\} \text {. }
$$

Which can easily be seen to be similar to the definition of the Hausdorff measure with one important difference; we need to to take the second infimum (5.1) to guarantee the measure is countably stable.

We note that there are topological conditions that can help us avoid taking the second infimum. Recall that $\operatorname{dim}_{P}(F)=\overline{\operatorname{dim}}_{B}(F)$ if $F$ is compact and $\overline{\operatorname{dim}}_{B} F \cap O=\overline{\operatorname{dim}}_{B} F$ for every open set $O$ that intersects $F$ non-trivially, see [Fal14, Corollary 3.10]. Similarly, we can prove the following Lemma.

Lemma 5.2. Let $\mathbb{L}$ be a family of IFS and let $\mathbb{P}$ be a random code-tree measure with associated attractor $F_{\tau}$. Let $h(t)$ be a doubling gauge function and assume that all maps $f_{\lambda}^{i} \in \mathbb{I}_{\lambda} \in \mathbb{L}$ are strict contractions such that there exist $0<c_{\min } \leq c_{\max }<1$ such that

$$
c_{\min }|x-y| \leq\left|f_{\lambda}^{i}(x)-f_{\lambda}^{i}(y)\right| \leq c_{\max }|x-y|
$$

for all $\lambda \in \Lambda$ and $i$ and all $x, y \in \mathbb{R}^{d}$. Let $\tau \in \mathcal{T}$, then

$$
\mathscr{P}_{0}^{h}\left(F_{\tau}\right)=\infty \quad \Longrightarrow \quad \mathscr{P}^{h}\left(F_{\tau}\right)=\infty
$$

and

$$
\mathscr{P}_{0}^{h}\left(F_{\tau}\right)=0 \quad \Longrightarrow \quad \mathscr{P}^{h}\left(F_{\tau}\right)=0 .
$$

Note that we did not make any assumption on the contractions and separation conditions in this Lemma.

Proof. Equation (5.3) follows from the definition of $\mathscr{P}^{h}$ and it remains to show (5.2), i.e. we need to show that

$$
\inf \left\{\sum_{i=1}^{\infty} \mathscr{P}_{0}^{h}\left(E_{i}\right) \mid F_{\tau} \subseteq \bigcup_{i=1}^{\infty} E_{i}\right\}=\infty
$$

if $\mathscr{P}_{0}^{h}\left(F_{\tau}\right)=\infty$. Now $F_{\tau}$ is compact, and so we can assume that $\left\{E_{i}\right\}$ is finite. Thus there exists $k$ and $j$ such that there exists $v_{j} \in \Sigma_{N_{k}(\tau)}$ with $f_{\mathbf{T}_{\tau}\left(v_{j}\right)}\left(F_{\sigma^{v_{j}} \tau}\right) \subset E_{j}$. So, for some $n$ dependent on the cover,

$$
\mathscr{P}^{h}\left(F_{\tau}\right)=\inf \left\{\sum_{i=1}^{n} \mathscr{P}_{0}^{h}\left(E_{i}\right) \mid F_{\tau} \subseteq \bigcup_{i=1}^{n} E_{i}\right\}
$$




$$
\begin{aligned}
& \geq \inf \left\{\mathscr{P}_{0}^{h}\left(E_{j}\right) \mid F_{\tau} \subseteq \bigcup_{i=1}^{n} E_{i}\right\} \\
& \geq \inf \left\{\mathscr{P}_{0}^{h}\left(f_{\mathbf{T}_{\tau}\left(v_{j}\right)}\left(F_{\sigma^{v_{j}}}\right)\right) \mid F_{\tau} \subseteq \bigcup_{i=1}^{n} E_{i}\right\} \\
& \geq \inf \left\{\lim _{\delta \rightarrow 0} \kappa \mathscr{P}_{\delta}^{h}\left(F_{\sigma^{v_{j}}}\right) \mid F_{\tau} \subseteq \bigcup_{i=1}^{n} E_{i}\right\}=\infty, \quad \text { (a.s.) }
\end{aligned}
$$

where the infimum is taken over all finite covers and $\kappa$ is a finite constant arising from the maximal distortion of the map $f($.$) (bounded by c_{\min }^{N_{k}}$ and $c_{\max }^{N_{k}}$ ) and the doubling of $h$.

\subsection{Bounds for equicontractive RIFS}

Inspired by the recent progress on the packing measure of random recursive attractors mentioned above, we would hope that using the gauge $h_{1}(t, \beta, \gamma)$ should give similar similar convergence and divergence, depending on the sign of $\gamma$. This can be achieved by considering the natural dual to $h_{1}$. Let $s \geq 0, \gamma \in \mathbb{R}$ and $\beta>0$, we set

$$
h_{1}^{*}(t, \beta, \gamma)=t^{s} \exp (-\sqrt{2 \beta \log (1 / t) \log \log (\beta \log (1 / t))})^{1-\gamma} .
$$

We remark that, in light of Lemma 5.2, we only sketch proofs.

Theorem 5.3. Let $F_{\omega}$ be the random homogeneous attractor associated to the self-similar RIFS $(\mathbb{L}, \mu)$ satisfying the UOSC and suppose that $c_{\lambda}^{i}=c_{\lambda} \in\left[c_{\min }, c_{\max }\right]$ for every $i \in$ $\left\{1, \ldots, \# \mathbb{I}_{\lambda}\right\}$ and $\lambda \in \Lambda$, where $0<c_{\min } \leq c_{\max }<1$. Let $\varepsilon>0, s=\operatorname{ess}_{\operatorname{dim}_{H}} F_{\omega}=$ ess $\operatorname{dim}_{P} F_{\omega}$ and $\beta_{0}^{*}=\eta_{0} \operatorname{Var}\left(\log \mathfrak{S}_{\omega_{1}}^{s}\right)$ for some $\eta_{0}^{*} \in \mathbb{R}$ (arising in the proof). Then $\mathscr{P}_{1}^{*}\left(t, \beta_{0}^{*}, \varepsilon\right)\left(F_{\omega}\right)=\infty$ almost surely.

Proof. By Lemma 5.2 we only have to analyse $\lim _{\delta \rightarrow 0} \mathscr{P}_{\delta}^{h_{1}^{*}\left(t, \beta_{0}^{*}, \varepsilon\right)}\left(F_{\omega}\right)$. Let $\langle X\rangle$ denote the compact convex hull of $X$. Since $c_{\lambda}$ is uniformly bounded away from 0 and 1 and $\sup _{\lambda \in \Lambda} \# \mathbb{I}_{\lambda}<\infty$ there exist $l$ and there exists at least one $e_{c h}(\omega) \in \mathbf{C}_{\omega}^{l}$ for which we have $f_{e_{c h}(\omega)}\left(\left\langle F_{\omega}\right\rangle\right) \subset\left\langle F_{\omega}\right\rangle$. Thus we get, in a similar fashion to the Hausdorff measure argument,

$$
\begin{aligned}
\lim _{\delta \rightarrow 0} \mathscr{P}_{\delta}^{h_{1}^{*}\left(t, \beta_{0}^{*}, \varepsilon\right)}\left(F_{\omega}\right) & =\lim _{\delta \rightarrow 0} \sup \left\{\sum_{i=1}^{\infty} h_{1}^{*}\left(2 r_{i}, \beta_{0}^{*}, \varepsilon\right) \mid\left\{B\left(x_{i}, r_{i}\right)\right\}\right. \text { is a disjoint } \\
\text { collection of balls with } \left.2 r_{i}<\delta \text { and } x_{i} \in F_{\omega}\right\} & \\
& \geq \limsup _{k \rightarrow \infty} \sum_{e \in \mathbf{C}_{\omega}^{k}} h_{1}^{*}\left(\left|f_{e e_{c h}\left(\sigma^{k} \omega\right)}\left(F_{\sigma^{k+l}}\right)\right|, \beta_{0}^{*}, \varepsilon\right) \\
& \geq \limsup _{k \rightarrow \infty}\left(\prod_{i=1}^{k} \mathcal{N}_{\omega_{i}}\right) h_{1}^{*}\left(c_{\omega_{1}} c_{\omega_{2}} \ldots c_{\omega_{k}} c_{\min }^{l}, \beta_{0}^{*}, \varepsilon\right) \\
& \geq \limsup _{k \rightarrow \infty}\left(\prod_{i=1}^{k} \mathcal{N}_{\omega_{i}}\right) \kappa\left(c_{\omega_{1}} c_{\omega_{2}} \ldots c_{\omega_{k}}\right)^{s} \exp (-(1-\varepsilon) \\
& \left.\cdot \sqrt{\beta_{0}^{*} \log \left(1 /\left(c_{\omega_{1}} \ldots c_{\omega_{k}}\right) \log \log \left(\beta_{0}^{*} \log \left(1 /\left(c_{\omega_{1}} \ldots c_{\omega_{k}}\right)\right)\right.\right.}\right)
\end{aligned}
$$




$$
\begin{aligned}
& \geq \limsup _{k \rightarrow \infty} \kappa \exp \left(\sum_{i=1}^{k} \log \mathfrak{S}_{\omega_{i}}^{s}-(1-\varepsilon) \sqrt{v k \log \log v k}\right) \\
& =\infty
\end{aligned}
$$

writing $v=\operatorname{Var}\left(\mathfrak{S}_{\omega_{1}}^{s}\right)$ and having used the law of the iterated logarithm in the last step.

Finally, we also obtain an upper bound.

Theorem 5.4. Let $F_{\omega}$ be the random homogeneous attractor associated to the self-similar $\operatorname{RIFS}(\mathbb{L}, \mu)$ satisfying the UOSC and suppose that $c_{\lambda}^{i}=c_{\lambda} \in\left[c_{\min }, c_{\max }\right]$ for every $i \in$ $\left\{1, \ldots, \# \mathbb{I}_{\lambda}\right\}$ and $\lambda \in \Lambda$, where $0<c_{\min } \leq c_{\max }<1$. Let $\varepsilon>0, s=\operatorname{ess}_{\operatorname{dim}_{H}} F_{\omega}=$ $\operatorname{ess} \operatorname{dim}_{P} F_{\omega}$ and $\beta^{*}=\eta \operatorname{Var}\left(\log \mathfrak{S}_{\omega_{1}}^{s}\right)$ for some $\eta^{*} \in \mathbb{R}$ (arising in the proof), then

$$
\mathscr{P}^{h_{1}^{*}\left(t, \beta^{*}, \varepsilon\right)}\left(F_{\omega}\right)=0
$$

holds almost surely.

Proof. By the homogeneity of the construction

$$
\begin{array}{r}
\sup \left\{\sum_{i=1}^{\infty} h_{1}^{*}\left(2 r_{i}, \beta_{0}^{*}, \varepsilon\right) \mid\left\{B\left(x_{i}, r_{i}\right)\right\} \text { are } \text { disjoint balls with } 2 r_{i}<\delta \text { and } x_{i} \in F_{\omega}\right\} \\
\leq \kappa \sup _{n \geq k(\delta)}\left\{\left(\prod_{i=1}^{n} \mathcal{N}_{\omega_{i}}\right) h_{1}^{*}\left(c_{\omega_{1}} \ldots c_{\omega_{n}}, \beta^{*}, \varepsilon\right)\right\}
\end{array}
$$

for some $\kappa>0$ depending on the diameter of $F_{\omega}$ and the doubling properties of $h_{1}$ only. So, for an appropriately chosen $\eta$, we obtain the desired conclusion from the law of the iterated logarithm.

\subsection{Existence of a gauge function}

Lemma 5.2 is unfortunately not sufficient to allow us to prove the non-existence of a gauge function with positive and finite packing measure using the same approach as in Section 4. However, the underlying idea still holds as the packing measure should, intuitively behave like

$$
\limsup _{k \rightarrow \infty} \sum_{e \in \mathbf{T}_{\tau}^{k}} h\left(c_{e}\right) .
$$

We therefore conjecture

Conjecture 5.5. Let $\mathbb{L}$ be a family of IFS that satisfy the UOSC and Condition 2.2. Let $\mathbb{P}$ be a random code-tree measure and assume that $F_{\tau}$ is not almost deterministic. Further, let $h(t)$ be any gauge function. Then,

$$
\mathbb{P}\left\{\tau \in \mathcal{T} \mid \mathscr{P}^{h}\left(F_{\tau}\right) \in\{0, \infty\}\right\}=1 .
$$

In particular, there does not exist a gauge function that gives positive and finite measure almost surely.

\section{Implications for a random implicit theorem}

There are two notable implications that our result has for random attractors in general. One concerns a random analogue of the implicit theorem due to Falconer [Fal89], whereas the other concerns the question on whether $V$-variable models interpolate between random homogeneous and random recursive sets. 


\subsection{The implicit theorems}

The implicit theorems are two statements about metric spaces that give a checkable condition for the set to have equal Hausdorff and upper-box counting dimension. Further, they give sufficient conditions for positive and finite Hausdorff measure. Both appeared first in Falconer [Fal89] but can also be found as [Fal97, Theorems 3.1 and 3.2].

Proposition 6.1. Let $F$ be a non-empty subset of $\mathbb{R}^{d}$ and let $a>0$ and $r_{0}>0$. Write $s=\operatorname{dim}_{H} F$ and suppose that for every set $U$ that intersects $F$ such that $|U|<r_{0}$ there is a mapping $g: U \cap F \rightarrow F$ with

$$
a|x-y| \leq|U| \cdot|g(x)-g(y)|
$$

for every $x, y \in F$. Then, $\mathscr{H}^{s}(F) \geq a^{s}>0$ and the upper box-counting dimension of $F$ coincides with $s$.

Heuristically, this means that if every small enough piece of a set $F$ can be embedded into the entire set $F$ without 'too much distortion', the Hausdorff measure is positive and all the commonly considered dimensions such as Hausdorff, packing, and box-counting dimension, coincide. Similarly, the second implicit theorem is.

Proposition 6.2. Let $F$ be a non-empty compact subset of $\mathbb{R}^{d}$ and let $a>0$ and $r_{0}>0$. Write $s=\operatorname{dim}_{H} F$ and suppose that for every closed ball $B$ with centre in $F$ and radius $r<r_{0}$ there exists a map $g: F \rightarrow B \cap F$ satisfying

$$
a r|x-y| \leq \mid g(x)-g(y 0 \mid
$$

for all $x, y \in F$. Then $\mathscr{H}^{s}(F) \leq 4^{s} a^{-s}<\infty$ and the upper box-counting dimension is $s$.

Here, the intuitive picture is that every ball centred in $F$ contains a 'not too small' copy of the entire set $F$. We remark that the second theorem can be applied to all self-similar and self-conformal sets and thus we conclude that their box-counting and Hausdorff dimensions coincide regardless of any overlap conditions. Further, we can conclude that their Hausdorff measure is always finite. If we additionally have overlap conditions such as the open set condition, we can apply the first implicit theorem and get not just finite but also positive measure.

It has been a long-standing question whether there exists some random analogue of such statement. It is certainly feasible that such a statement can exist, as it is known that the Hausdorff and box-counting dimensions agree for many common random models such as random recursive, $V$-variable, and also graph directed models, see [Tro17b] and references therein.

However, a general statement for random sets that includes a conclusion of positive and finite measure has been more elusive. It was known for a while that the Hausdorff measure of random recursive sets is 0 almost surely for reasonable random self-similar sets, as we have discussed in Section 2. Any potential implicit theorems with results on the positivity of the Hausdorff measure must have taken into account the underlying process and would have been associated with a gauge function for that process.

Our results show, however, that even though random homogeneous sets are very natural and should surely have come under the scope of such a theorem, the non-existence of a gauge functions means that there could not be such a general implicit statement.

The best one could hope for for such an implicit theorem is just a statement about the coincidence of Hausdorff and box-counting dimension, i.e. that it does not matter whether one takes the infimum over all coverings, but restricts oneself to coverings with sets of equal diameter. 


\section{2 $V$-variable interpolation}

The $V$-variable model was first introduced to interpolate between the random homogeneous and the random recursive process. It was suggested in Barnsley et al. [BHS12] that the Hausdorff dimension of (reasonably picked) $V$-variable sets should interpolate between the two models. That is, let $F_{V}$ be the random set created by a $V$-variable process sharing the same RIFS $(\mathbb{L}, \mu)$. Further, denote by $F_{\infty}$ the random attractor of the associated random recursive set. Barnsley et al. claim in [BHS12] that ess $\operatorname{dim}_{H} F_{V} \rightarrow \operatorname{ess} \operatorname{dim}_{H} F_{\infty}$ as $V \rightarrow \infty$ but only support this with some computational evidence. As far as we are aware, there is no known proof that the dimension converges. What is more, it is not even known whether this sequence of dimensions is increasing. The computational evidence seems to suggest the following conjecture.

Conjecture 6.3. Let $(\mathbb{L}, \mu)$ be a RIFS that satisfies the UOSC and Condition 2.2. Let $F_{V}$ be the associated $V$-variable, and $F_{\infty}$ be the random recursive attractor. Let $D: \mathbb{N} \cup\{\infty\} \rightarrow \mathbb{R}^{+}$ be given by $D(V)=\operatorname{ess}^{\operatorname{dim}_{H}} F_{V}$ and suppose that $D(1)<D(\infty)$. Then $D(V)$ is strictly increasing and $D(V) \rightarrow D(\infty)$ as $V \rightarrow \infty$.

One could further ask whether there is a closed form expression for the Hausdorff dimension of equicontractive RIFS $V$-variable sets, as there is for random homogeneous and random recursive: $\mathbb{E}\left(\log \mathcal{N}_{\lambda}\right) / \log c$ and $\log \mathbb{E}\left(\mathcal{N}_{\lambda}\right) / \log c$, respectively.

However, our work has shown that $V$-variable sets have much more in common with random homogeneous processes than with the random recursive. In both of the former there simply cannot be a gauge function that adequately describes the fine dimension, whereas there is one for the latter. This implies that $V$-variable processes cannot interpolate the fine dimension (as there is nothing to interpolate with), but they could still interpolate in the coarse sense.

\section{Acknowledgements}

The work was started while the author attended the ICERM semester programme Dimension Theory and Dynamics in the spring of 2016 and the author thanks ICERM and Brown University for their financial support. Further, ST was at initially at the University of St Andrews and supported by EPSRC Doctoral Training Grant EP/K503162/1 and later by the Faculty of Mathematics at the University of Waterloo and NSERC grants 2016-03719 and RGPIN-2014-03154. The author is indebted to Kenneth Falconer, Mike Todd, Jonathan Fraser, and Julius Jonušas for many fruitful discussions.

\section{References}

[AL06] K. B. Athreya and S. N. Lahiri. Measure theory and probability theory. Springer Texts in Statistics. Springer, New York, 2006.

[AN72] K. B. Athreya and P. E. Ney. Branching processes. Springer-Verlag, New YorkHeidelberg, 1972. Die Grundlehren der mathematischen Wissenschaften, Band 196.

[Ber03] A. Berlinkov. Exact packing dimension in random recursive constructions. Probab. Theory Related Fields, 126, (2003), 477-496.

[BHS05] M. Barnsley, J. Hutchinson, and Ö. Stenflo. A fractal valued random iteration algorithm and fractal hierarchy. Fractals, 13, no. 2, (2005), 111-146. 
[BHS08] M. F. Barnsley, J. E. Hutchinson, and Ö. Stenflo. $V$-variable fractals: fractals with partial self similarity. Adv. Math., 218, no. 6, (2008), 2051-2088.

[BHS12] M. Barnsley, J. E. Hutchinson, and Ö. Stenflo. $V$-variable fractals: dimension results. Forum Math., 24, no. 3, (2012), 445-470.

[BM02] A. Berlinkov and R. D. Mauldin. Packing measure and dimension of random fractals. Journal of Theoretical Probability, 15, (2002), 695-713.

[Fal86] K. J. Falconer. Random fractals. Math. Proc. Cambridge Philos. Soc., 100, (1986), 559-582.

[Fal89] K. J. Falconer. Dimensions and measures of quasi self-similar sets. Proc. Amer. Math. Soc., 106, no. 2, (1989), 543-554.

[Fal97] K. Falconer. Techniques in fractal geometry. John Wiley \& Sons Ltd., Chichester, 1997.

[Fal14] K. Falconer. Fractal geometry. John Wiley \& Sons, Ltd., Chichester, 3rd edition, 2014. Mathematical foundations and applications.

[Fre10] U. R. Freiberg. Some remarks on the Hausdorff and spectral dimension of $V$ variable nested fractals. In Recent developments in fractals and related fields, Appl. Numer. Harmon. Anal., pp. 267-282. Birkhäuser Boston, Inc., Boston, MA, 2010.

[Fur14] H. Furstenberg. Ergodic theory and fractal geometry, volume 120 of CBMS Regional Conference Series in Mathematics. American Mathematical Society, Providence, RI, 2014.

[GMW88] S. Graf, R. D. Mauldin, and S. C. Williams. The exact Hausdorff dimension of random recursive constructions. Memoirs of the American Mathematical Society, 71.

[Gra87] S. Graf. Statistically self-similar fractals. Probab. Theory Related Fields, 74, no. 3, (1987), 357-392.

[Ham92] B. M. Hambly. Brownian motion on a homogeneous random fractal. Probab. Theory Related Fields, 94, no. 1, (1992), 1-38.

[Hat00] K. Hattori. Exact Hausdorff dimension of self-avoiding processes on the multidimensional Sierpinski gasket. J. Math. Sci. Univ. Tokyo, 7, no. 1, (2000), $57-98$.

[Hu95] X. Hu. The measure functions of random Cantor set and fractals determined by subordinators. Chinese Science Bulletin, 40, no. 6, (1995), 441-445.

[Hu96] X. Hu. The exact packing measure for a random re-ordering of the Cantor set. Sci. China Ser. A, 39, no. 1, (1996), 1-6.

[JJK $\left.{ }^{+} 14\right] \quad$ E. Järvenpää, M. Järvenpää, A. Käenmäki, H. Koivusalo, O. Stenflo, and V. Suomala. Dimensions of random affine code tree fractals. Ergodic Theory Dynam. Systems, 34, no. 3, (2014), 854-875. 
[JJLS16] E. Järvenpää, M. Järvenpää, B. Li, and Ö. Stenflo. Random affine code tree fractals and Falconer-Sloan condition. Ergodic Theory Dynam. Systems, 36, (2016), 1516-1533.

[JJWW17] E. Järvenpää, M. Järvenpää, M. Wu, and W. Wu. Random affine code tree fractals: Hausdorff and affinity dimensions and pressure. Math. Proc. Cambridge Philos. Soc., 162, no. 2, (2017), 367-382.

[Liu96] Q. Liu. The exact Hausdorff dimension of a branching set. Probab. Theory Related Fields, 104, no. 4, (1996), 515-538.

[Liu00] Q. Liu. Exact packing measure on a Galton-Watson tree. Stochastic Process. Appl., 85, (2000), 19-28.

[MGW87] R. D. Mauldin, S. Graf, and S. C. Williams. Exact Hausdorff dimension in random recursive constructions. Proc. Natl. Acad. Sci. USA, 84, (1987), 39593961.

[Ols03] L. Olsen. The exact Hausdorff dimension functions of some Cantor sets. Nonlinearity, 16, (2003), 963-970.

[RU11] M. Roy and M. Urbański. Random graph directed Markov systems. Discrete Contin. Dyn. Syst., 30, no. 1, (2011), 261-298.

[Tro17a] S. Troscheit. Dimension theory of random self-similar and self-affine constructions. Ph.D. thesis, University of St Andrews, April 2017.

[Tro17b] S. Troscheit. On the dimensions of attractors of random self-similar graph directed iterated function systems. J. Fractal Geom., 4, no. 3, (2017), 257-303.

[Tro19] S. Troscheit. The quasi-Assouad dimension for stochastically self-similar sets (FirstView). Royal Society of Edinburgh Proceedings A, pp. 1-15.

[Wat07] T. Watanabe. Exact Hausdorff measure on the boundary of a Galton-Watson tree. Ann. Probab., 35, (2007), 1007-1038. 